\title{
Energetics of the Beamed Zombie Turbulence Maser Action Mechanism for Remote Detection of Submerged Oceanic Turbulence
}

\author{
Carl H. Gibson ${ }^{1}$, Valery G. Bondur ${ }^{2}$, R. Norris Keeler ${ }^{3}$ and Pak Tao Leung ${ }^{4}$ \\ ${ }^{1}$ Departments of Mechanical and Aerospace Engineering and Scripps Institution of Oceanography, \\ University of California at San Diego, La Jolla, CA, 92122-0411, USA \\ ${ }^{2}$ Aerocosmos Scientific Center of Aerospace Monitoring, Moscow, Russia \\ ${ }^{3}$ Directed Technologies, Inc., Arlington, VA \\ ${ }^{4}$ Department of Oceanography, Physical Section, Texas A\&M University, \\ College Station, TX 77843, USA \\ Email: cgibson@ucsd.edu
}

(Received February 7, 2006; accepted October 1, 2006)

\begin{abstract}
Sea surface brightness spectral anomalies from a Honolulu municipal outfall have been detected from space satellites in $200 \mathrm{~km}^{2}$ areas extending $20 \mathrm{~km}$ from the wastewater diffuser (Leung and Gibson 2004, Bondur 2005, Keeler et al. 2005, Gibson et al. 2006). Dropsonde and towed body microstructure measurements show greatly enhanced viscous and temperature dissipation rates above the outfall trapping-layer. Fossil-turbulencewaves (FTWs) and secondary zombie-turbulence-waves (ZTWs) break as they propagate near-vertically and then break again near the surface to produce wind-ripple smoothing with narrow-wavelength $\lambda$ patterns from the soliton-like internal waves that supply turbulence energy to advected outfall fossils and to the ZTWs they radiate. The $\lambda=30-250 \mathrm{~m}$ solitons reflect an efficient maser-action conversion of horizontal tidal and current kinetic energy by bottom boundary layer turbulence events to near-vertical FTWs with $\lambda$ the Ozmidov scale of the events at fossilization. Secondary (zombie) turbulence amplifies, channels in chimneys, and near-vertically beams ambient internal wave energy at scales $\lambda$ just as energized metastable molecules amplify and beam quantum wavelengths in astrophysical lasers and masers around stars. Kilowatts of buoyancy power from the treatment plant produce fossil turbulence patches trapped below the thermocline. Beamed zombie turbulence maser action (BZTMA) in mixing chimneys amplifies these kilowatts into the megawatts of surface turbulence dissipation required to affect brightness on wide sea surface areas. The BZTMA vertical mixing mechanism appears critical to vertical oceanic transport of information, heat, mass and momentum, and to the conversion of barotropic tides to baroclinic tides.
\end{abstract}

Keywords: Turbulence, diffusion, and mixing processes, Fine structure and microstructure, Nearshore processes, Remote sensing.

\section{INTRODUCTION}

The Remote Anthropogenic Sensing Program (RASP) is a continuing international effort to test, understand and quantify remote detection of submerged turbulence and fossil turbulence in the ocean by optical and radar space satellite images, Bondur and Savin (1995), Bondur and
Filatov (2003), Leung and Gibson (2004), Keeler et al. (2004), Wolk et al. (2004), Bondur (2005), Keeler, Bondur and Gibson (2005, KBG), Gibson et al. (2006: GBKL). The largest Honolulu, Hawaii, municipal wastewater outfall, from Sand Island, provides a relatively steady source of submerged turbulence, trapped and fossilized by 
buoyancy forces of the stably stratified receiving waters in Mamala Bay, Fisher et al. (1997).

Surface smoothing, plus the enhanced turbulence and temperature dissipation rates and hydrodynamic phase diagrams observed by microstructure profiling above the outfall diffuser, prove that the trapped turbulence and fossil turbulence produce near-vertical radiation of unstable internal waves that mix and cause detectable surface effects. Thousands of hydrodynamic phase diagrams of vertically profiled microstructure patches prove that outfall fossil turbulence patches are significantly more powerful and have larger sizes at the beginning of their fossilization than fossil turbulence patches from ambient sources. Few outfall turbulence patches were found in their fully active turbulence state, proving that the fossil turbulence hydrodynamic state is more persistent.

Advected outfall fossil turbulence patches serve as sites for secondary turbulence events that near-vertically beam information about ambient soliton-like internal waves (SIWs) more efficiently than smaller ambient fossil turbulence patches. The narrow bandwidth and large $\lambda$ of the SIWs indicates their likely source is intermittent bottom topography fossil turbulence.

Vertical beaming is by internal wave maser action, where a large fraction of the stratified bottom turbulence kinetic energy is beamed near-vertically as breaking fossil turbulence waves (FTWs) that form ZTW chimneys. It is not true that waves do not mix and that internal waves always cascade from large scales to small. Linear waves do not mix much, but FTWs mix very effectively and radiate nonlinear internal waves that mix by producing turbulence and even larger nonlinear internal waves such as internal tides and solitary waves.

In particular, from the present measurements it appears likely that barotropic tidal waves produce baroclinic internal tides more by tidal driven turbulent maser-action mixing and turbulent drag at small scales on the rough topography than directly at large scales by form drag, Petrelis et al. (2006). Internal tides then distribute the tidal power broadly in the ocean interior, Rainville and Pinkel (2006).

Baroclinic internal tidal waves have been detected $1700 \mathrm{~km}$ from their source, Lozovatski et al. (2003). Internal tidal waves from an Atlantic Ridge seamount $3 \mathrm{~km}$ deep, as well as the seamount, are clearly visible at the sea surface, GBKL (Fig. 1c). Internal waves supply energy to secondary fossil turbulence events that use part of the energy for local mixing and beam the rest near-vertically to the sea surface, mixing on the way, Gibson $(1980,1986,1996)$. From satellite measurements, up to $35 \%$ of $\sim 10^{15}$ watts of surface tidal energy is dissipated in the deep ocean, Egbert and Ray (2003).

The tidal conversion process is illustrated in Fig. 1a, modified from Fig. 2 of Rudnick et al. (2003) that discusses the Hawaiian Ocean Mixing Experiment (HOME) and preliminary results of the Levine and Boyd (2006) moored study on the Keana Ridge. HOME investigators hypothesize a cascade of barotropic tidal energy directly from topography to the baroclinic internal tide and a monotonic internal wave energy cascade to smaller scales.
Finally, turbulence is produced when the smallest internal waves break, Pinkel and Rudnick (2006) and papers in their Edited Volume 36 of JPO. This internal wave cascade from large to small scales is employed by Garrett (2003) to support of the surprising claim of Gregg et al. (2003) that turbulence dissipation rates and mixing at the equator are minimal rather than maximal as shown by Baker and Gibson (1987) and Gibson (1991b). Equatorial waters are highly productive biologically despite maximum stratification by solar radiation and rainfall, which makes the Gregg et al. (2003) claim surprising and the Garrett (2003) internal wave cascade from large scales to smaller scales to turbulence also questionable.

A new alternative hypothesis for tidal conversion is suggested from the RASP observations in Fig. 1a, where the $300 \mathrm{~m}$ vertical temperature oscillations over the Hawaiian ridge are produced directly by semi-diurnal period $(12.4 \mathrm{~h})$ bottom turbulence events, fossil turbulence, and fossil-turbulence-wave (FTW) radiation. FTW small-scale internal waves break and mix as they propagate nearly vertically leaving fossil turbulence patches that serve as sites for further breaking.

Secondary turbulence events on fossil turbulence patches (zombies) also fossilize and radiate more small-scale internal waves (ZTWs) near-vertically in mixing chimney beams. Thus the tidal energy cascade is from barotropic tides directly to bottom turbulence events that make smallscale near-vertically propagating internal waves that make large-scale horizontal internal waves that make turbulence that makes small-scale near-vertical internal waves, etc. The complex process was termed "big bangs and continuous creation” by Gibson (1987). Turbulence is the cause of internal tides, not the effect.

Large amplitude internal waves are observed where tidal currents flow over shelf-breaks, banks, and sills and under river plumes. These waves are assumed to be directly radiated, but may also reflect large amplitude vertical mixing from turbulence, fossil turbulence, fossil turbulence waves, and the BZTMA vertical mixing mechanism. Figure $1 \mathrm{~b}$ shows tidal internal waves radiated from the Columbia River plume front, Nash and Moum (2005). Turbulent mixing occurs at the sea-water riverwater front formed when the incoming tide meets the outgoing river plume. Fossil turbulence waves extract energy from the shear turbulence and beam it downward by maser action, breaking, mixing, and deepening the mixed region height $h$ below the front. When the tidal velocity $u$ decreases below the linear internal wave speed $c \approx \sqrt{g \Delta \rho h / \rho}$, internal wave packets are released and propagate away from the front.

In the RASP 2002, 2003 and 2004 experiments, parachute drogues released near the wastewater trapping depth at the diffuser tracked the complex advection of outfall fossil turbulence patches by ambient currents. These 6-10 m vertical scale patches persist for many days and are critical to the remote detection of the outfall turbulence, the remote detection of the intermittent bottom turbulence events, and visualization of the bottom itself, GBKL. Several ships, buoys, and bottom-mounted instruments 
Carl H. Gibson et al. / JAFM , Vol. 1, No. 1, pp. 11-42, 2008.

were deployed to provide environmental information. Dropsonde vertical and horizontally towed sensors provide synoptic microstructure information.

Only one panchromatic image was available for RASP 2002 from an optical satellite, but multispectral and radar images from a variety of satellites were collected in 2003 and 2004. Optical and radar satellite images of sea surface brightness have been analyzed for anomalies using random spectral phase and texture methods, Bondur (2005). Outfall anomalies to distances of $45 \mathrm{~km}$ have now been detected by several radar satellites and multispectral optical satellites. In the present paper we focus on SAR (synthetic aperture radar) and panchromatic optical detections in RASP 2003 compared to sea truth microstructure measurements and the fluid mechanical energetic constraints on physical models required to explain these remarkable and unexpected results.

RASP 2002 results are given by Bondur (2004, 2005), Bondur and Filatov (2003), Leung and Gibson (2004), KBG (2005) and GBKL (2006). In RASP 2002, microstructure sea truth measurements were confined to distances less than $3 \mathrm{~km}$ from the diffuser because it was not anticipated that surface anomalies induced by the wastewater injection would extend much beyond the $\sim 0.1 \mathrm{~km}^{2}$ area of active turbulence and fossil turbulence waves produced directly by the waste water diffuser.

Optical surface brightness anomalies were detected in two lobes from the September 2, 2002, Ikonos-2 satellite image covering $70 \mathrm{~km}^{2}$ and extending $10 \mathrm{~km} \mathrm{SW}$ and $7 \mathrm{~km} \mathrm{SE}$, Bondur and Filatov (2003). Larger and smaller areas of detection in similar locations were found from RASP 2003 optical images September 2, 13 and 14, and RADARSAT and EVISAT radar images Sept. 11 and Sept. 13, 2003, Bondur (2004). These are discussed and compared to microstructure data in the present paper, GBKL and KBG.

We suggest the optical spectral anomaly detection requires a beamed zombie turbulence maser action (BZTMA) mixing chimney mechanism. BZTMA is analogous to beamed astrophysical masers that strongly amplify the radiated power of stars at low maser frequencies by activating metastable maser molecules such as $\mathrm{OH}^{-}$(water missing a proton), KBG and GBKL. Power levels in narrow radio frequency bands are amplified by factors $>10^{6}$ by such masers, making star formation regions and galaxy cores detectable that are obscured by dust at optical frequencies, Cernicharo et al. (2006).

In the RASP experiments, optical sea surface brightness spectral anomalies are detected as narrow-spectralfrequency Fourier elements with wavelengths $30-220 \mathrm{~m}$ that are weak or missing from ambient background sea surface areas.

According to BZTMA, internal waves produced by stratified turbulence are near saturation at the inertialvortex-buoyancy transition of turbulence to fossil turbulence and radiate internal waves nearly vertically as fossil turbulence waves (FTWs). The waves are narrow frequency band because they are produced by fossil turbulence in a narrow range of length scales; that is, near the Ozmidov length scale at the beginning of fossilization
$L_{R_{0}}$, Gibson (1980).

For a bottom boundary layer event with a $220 \mathrm{~m}$ overturn scale and $\mathrm{N} \sim 5 \times 10^{-3} \mathrm{~s}^{-1}$ at $350 \mathrm{~m}$ depth, the dissipation rate at beginning of fossilization $\varepsilon_{0}$ is $\sim 1.8 \times 10^{-2} \mathrm{~m}^{2} \mathrm{~s}^{-3}$ with $\varepsilon_{F} \sim 7.5 \times 10^{-9} \mathrm{~m}^{2} \mathrm{~s}^{-3}$, compared to $\varepsilon_{0} \sim 2.4 \times 10^{-3}$ $\mathrm{m}^{2} \mathrm{~s}^{-3}$ for a $10 \mathrm{~m}$ overturn at the pycnocline with $\mathrm{N} \sim$ $2 \times 10^{-2} \mathrm{~s}^{-1}$ and $\varepsilon_{F} \sim 1.2 \times 10^{-7} \mathrm{~m}^{2} \mathrm{~s}^{-3}$. These sample calculations give $\operatorname{Re}_{0} / \operatorname{Re}_{\mathrm{F}}$ values $2.4 \times 10^{6}$ and $2.0 \times 10^{4}$ that are close to measured Reynolds number ratios indicated by hydrodynamic phase diagrams for the observed microstructure patches (see Fig. 16 below).

Vertical mixing processes in the ocean (and the remote detection of submerged turbulence) are dominated by rare and powerful events that are intermittent in time, patchy in space, and difficult to adequately sample, Baker and Gibson (1987). Oceanic microstructure studies that ignore the intrinsic nonlinearity and consequent extreme spacetime intermittency of stratified turbulence are subject to large undersampling errors that may be qualitative as well as quantitative, as suggested for the Gregg et al. (2003) result.

In the following, we compare BZTMA theory and energetics to a direct internal wave radiation model and then discuss zombie turbulence formation theory and its reflection in the hydrodynamic phase diagram (HPD) method to evaluate microstructure patch hydrodynamic states, Gibson $(1980,1986)$. We then review the RASP 2003 satellite anomaly detections compared with vertical and horizontal microstructure observations. A discussion of results is presented along with conclusions.

\section{PHYSICAL PROCESSES AND ENERGETICS}

Turbulence is defined as an eddy-like state of fluid motion where the inertial vortex forces $\vec{v} \times \vec{\omega}$ of the eddies are larger than any of the other forces that tend to damp the eddies out, Gibson (1999). Because the momentum and energy transport mechanism is identical at all inertial scales and depends on the $\vec{v} \times \vec{\omega}$ force between eddies, a physical basis exists for the universal similarity laws of turbulence and turbulent mixing in the inertial range, Gibson (1991a).

Irrotational flows are by definition nonturbulent because the vorticity $\vec{\omega}$ of such flows is everywhere near zero. The turbulence energy cascade is always from small scales where turbulence and vorticity are produced to larger scales where the energy is stored or radiated. Turbulence can induce a cascade of kinetic energy from large scales to small in irrotational flows, but this is, by definition, a nonturbulent cascade.

It is often claimed that turbulence cascades from large scales to small; eg, Ferziger (2005). Such claims are unfortunate, erroneous, misleading, and widely believed. When turbulence is found cascading from small scales to 
large, as it clearly does for two-dimensional turbulence, it is commonly called an inverse cascade. Observations reveal that three dimensional turbulent jets, wakes, mixing layers, and boundary layers also grow from small scales to large. It is illogical to call such flows inverse cascades.

Winds and astronomical tides produce turbulence at the upper and lower boundaries of the ocean and the turbulent kinetic energy cascades to larger and larger scales by producing horizontal turbulence and currents and nearvertically propagating internal waves. For internal waves, propagation at any angle within $60^{\circ}$ of vertical is considered near-vertical or simply vertical. A typical angle of FTW propagation is $45^{\circ}$ because the horizontal velocity of turbulence generation matches the vertical velocity of FTW radiation. These small-scale internal waves are crucial to the vertical transport of information about submerged turbulence, internal waves and bottom topography, GBKL.

The physical processes of turbulence and vertical information transport in stably stratified fluids by turbulence driven internal-wave maser action are illustrated in Fig. 1c. Figure 1c (a) shows the interaction of adjacent vortices of the same sign that leads to merging and the turbulence cascade to large scales. Opposite sign eddy pairs also cause growth of the turbulent region by inducing entrainment. Figure 1c (b) shows the development of a bottom topography turbulence event radiating energy near vertically by internal wave maser action.

The turbulence driven vertical internal wave transport mechanism is called a maser action by analogy to quantum mechanical laser and maser actions, Leung and Gibson (2004), KBG and GBKL. In quantum mechanical masers an energy supply pumps ground state maser molecules to a higher metastable energy state. Radiation with photons matching this energy state stimulates decay to ground state with release of the maser photons in a cascade, amplifying the power in this frequency band by maser action. Beaming in solid state lasers is accomplished by carefully designed reflectors to create resonant cavities, Koechner (1999).

Because fossil turbulence waves are unstable by all the criteria developed in Sutherland (2001) they break as they propagate leaving a vertical trail of fossil turbulence patches. These patches mimic metastable maser molecules of quantum mechanical masers by extracting turbulence energy from SIW tilting motions, fossilizing, and radiating the energy near-vertically as secondary (zombie) turbulence waves. The trails can become channels termed BZTMA mixing chimneys analogous to beams formed in maser clouds around stars in astrophysical masers.

For the bottom turbulence event shown in Fig. 1c (b), turbulence begins at the Kolmogorov scale $L_{K}=\left(v^{3} / \varepsilon\right)^{1 / 4}$ after the boundary layer has grown to a thickness of about $5 L_{K}$. Inertial vortex forces $\vec{v} \times \vec{\omega}$ lift the eddies away from the boundary layer. The turbulence induces a large-scale to small-scale nonturbulent energy cascade in the external irrotational flow to $L_{K}$ viscous scales where the irrotational fluid becomes turbulent.

Mergers of same-spin eddies powers the turbulent energy cascade from viscous to energy scales. Such eddies induce inertial vortex $\vec{v} \times \vec{\omega}$ forces toward each other that cause them to merge as shown in Fig. 1c (a). Eddy pairing by $\vec{v} \times \vec{\omega}$ forces at all scales is the physical basis of the universal similarity laws of Kolmogorov. Nearby eddies of opposite spin have inertial vortex forces $\vec{v} \times \vec{\omega}$ away from each other and away from the turbulence zone. This causes propagation and expansion of the turbulent region by ingestion of ambient nonturbulent fluid.

Merging forces $\vec{v} \times \vec{\omega}$ of adjacent eddies with the same spin can also be understood as lift forces induced when vorticity of the eddies induces spin about each other. The speed over the sides facing the adjacent eddies is greater than that opposite, so the pressure is less by Bernoulli's equation. Think of weightless spinning baseballs curving around and toward each other. The merging behavior of adjacent vortex pairs has been frequently demonstrated in the laboratory, Munier and Leweke (2003), Driscoll et al. (2002).

Fossil turbulence is defined as a fluctuation in any hydrophysical field produced by turbulence that persists after the fluid is no longer turbulent at the scale of the perturbation. When the stratified boundary layer eddies of Fig. 1c (b) can no longer overturn by overcoming buoyancy forces, their turbulent kinetic energy is converted without loss to the energy of saturated internal waves termed fossil-vorticity-turbulence that bob without overturning at the ambient stratification frequency

$$
N=\left(g \rho^{-1} \partial \rho / \partial z\right)^{1 / 2} \text {. }
$$

Fossil turbulence microstructure patches (dashed line circles in Fig. 1c (b)) have vertical sizes proportional to the Ozmidov scale at fossilization $L_{R_{0}}=\left(\varepsilon_{0} / N^{3}\right)^{1 / 2}$, and serve as sites for secondary turbulence events (zombie turbulence), Gibson (1987). Fossil turbulence waves are ejected by inertial vortex forces near-vertically from the fossil turbulence patches at fossil turbulence velocities proportional to the shear velocity $\vec{v}$ for the boundary layer.

Figure 1d shows the RASP experiments (top), and summarizes the energetics of the BZTMA process (bottom). Municipal wastewater from the Sand Island treatment plant is pumped $2.4 \mathrm{~km}$ offshore to a 283 -jet $1040 \mathrm{~m}$ long diffuser pipe 70 meters deep. The hydraulic power $\mathrm{P}_{\mathrm{H}}$ required to pump this relatively low salinity wastewater to the ocean bottom at depth $h$ is the pressure difference between seawater and freshwater water columns $\delta \rho g h$ times the volumetric flow rate $V_{\text {rate }}$ of the effluent, where $g$ is gravity. For the present experiments $\left(\rho_{\mathrm{S}}-\rho_{\mathrm{W}}\right)$ is about $23 \mathrm{~kg} \mathrm{~m}^{-3}$, and the volume flow rate is 3-4 $\mathrm{m}^{3} \mathrm{~s}^{-1}$, giving hydraulic power $\mathrm{P}_{\mathrm{H}}=g h\left(\rho_{S^{-}} \rho_{W}\right) V_{\text {rate }} \sim 50$ kw. 
Observations near the outfall show strongly enhanced turbulence in the upper $10 \mathrm{~m}$ wind mixed layer with viscous dissipation rates $\varepsilon \sim 10^{-5} \mathrm{~m}^{2} \mathrm{~s}^{-3}$ compared to ambient values two orders of magnitude less, Gibson et al. (2006). Assuming turbulence produced by breaking zombie turbulence waves (ZTWs) is responsible for the observed brightness anomalies and if the surface turbulence from ZTW radiation were uniform with $\varepsilon \sim 10^{-5} \mathrm{~m}^{2} \mathrm{~s}^{-3}$ in the 70 $\mathrm{km}^{2}$ of surface layer $10 \mathrm{~m}$ deep for the RASP 2002 anomaly area then the dissipation power $P_{D}$ required for the remote detection would be 7 megawatts, about two orders of magnitude larger than the hydraulic power $\mathrm{P}_{\mathrm{H}}$ of the wastewater injection.

The ZTW radiation process is nonlinear and dissipative, with considerable breaking and energy loss due to shear layers and stratified layers on the way to the surface that can balance the lack of uniformity. Most of the outfall pumping power $\mathrm{P}_{\mathrm{H}}$ will be directed near-vertically as FTWs, leaving a small fraction for radiation of horizontal waves on the pycnocline that must somehow smooth the sea surface and permit remote detection at large distances in the anomaly areas. The RASP remote detection of outfall turbulence over areas up to $800 \mathrm{~km}^{2}$ requires sources for surface smoothing much more powerful than the $50 \mathrm{kw}$ outfall pump.

How much power is available to be extracted from ambient internal waves and re-radiated near-vertically by the zombie turbulence patch formation mechanism? For a rough $10^{2}$ $\mathrm{km}^{2}$ turbulent boundary layer area $A$ produced by the $0.5 \mathrm{~m}$ $\mathrm{s}^{-1}$ North Equatorial current with velocity $V$, the turbulent boundary layer power $\mathrm{P}_{\mathrm{T}}$ would be $\rho C_{D} V^{3} A=6 \times 10^{9} \mathrm{~kg}$ $\mathrm{m}^{2} \mathrm{~s}^{-2}$ or 6 gigawatts, taking the drag coefficient $C_{D}$ to be 0.5 .

The maximum power radiated near vertically $\left(\sim 45^{\circ}\right)$ as fossil turbulence waves for this area would therefore be $\sim 3$ gigawatts, using efficiencies up to $50 \%$ as found in laboratory experiments of Linden (1975). This is $10^{3}$ larger than our estimate of $\mathrm{P}_{\mathrm{D}}$ and $10^{6}$ larger than $\mathrm{P}_{\mathrm{H}}$. Tidal motions add to this BBL fossil turbulence wave energy supply, Munk and Wunsch (1998). Intermittency in the bottom topography and bottom speeds will decrease the power supply available, but fossilization of the surface breaking will preserve the patterns of bottom boundary layer FTWs imprinted, drawing on surface wave energy to keep them fresh and radiating to smooth the sea surface.

Linear internal waves propagate with directions and speeds determined by the frequency ratio $\omega / N$. As $\omega / N \rightarrow 1$ the propagation direction approaches vertical and the propagation speed approaches zero. High speed overturning eddies of stratified turbulence slow down as the turbulence patch grows and finally fossilize at the largest overturn scale $0.6 L_{R_{0}}$ at the stratification frequency $\mathrm{N}$. All of the turbulent kinetic energy is converted to saturated fossil vorticity turbulence internal waves (FVTs) that bob with frequency $\mathrm{N}$ and would not propagate at all if they were linear. Inertial-vortex forces couple FVTs to the stratification above and below the fossilized turbulence region creating FTWs that propagate near-vertically as large amplitude near-vertical Linden-Sutherland waves with vertical velocity matching the fossilization turbulence velocity, KBG (2005, Fig. 3).

Thus, all internal wave motions from the outfall should be radiated near vertically, and virtually no energy will be radiated horizontally. Whatever energy can emerge horizontally will be confined to pycnocline depths at least 40 to 50 meters below the sea surface, and will have little influence on the sea surface smoothing in patterns required for a remote detection of such directly radiated outfall waves. Soliton wave packets from extreme topographical turbulent bottom boundary layer (BBL) events will have much larger amplitude and variable directions as observed in spectral anomalies. They can interact with outfall fossil density turbulence patches advected offshore at near pycnocline depths to form zombie turbulence patches that produce the observed anomaly patterns according to the BZTMA mechanism of Fig. 1a by vertical radiation of ZTWs.

Figure 2 illustrates the fossil turbulence and zombie turbulence process. An actively turbulent patch (1) at the beginning of fossilization is shown at top left, with a generic vertical density profile reflecting the entrainment of increasing density differences as the active turbulence in the spherical patch grows in vertical scale. Without ambient energy input such patches enter the active-fossil regime (2), where the maximum Thorpe density displacement distance and ambient stratification frequency retain information about the initial viscous dissipation rate at fossilization $\varepsilon_{0}=3 L^{2} T_{\max } N^{3}$, but the patch $\varepsilon$ is less than $\varepsilon_{0}$.

The bobbing motions of the patch are radiated near vertically ( $\sim 5$ degrees) as fossil turbulence waves, shown as vertical arrows. Complete fossilization (3) occurs when $\varepsilon$ decreases to $\varepsilon_{\mathrm{F}}=30 v \mathrm{~N}^{2}$, where $v$ is the kinematic viscosity and $\mathrm{N}$ is the stratification frequency, Gibson (1980, p227). At complete fossilization with $\varepsilon=\varepsilon_{\mathrm{F}}$, buoyancy, viscous and inertial-vortex forces are equal and overturning of eddies within the patch ceases. Froude and Reynolds numbers are at their critical values.

The zombie turbulence formation process from fossils is illustrated by dashed arrows and the three patches (3), (4), (5) in Fig. 2. If the fossil density turbulence patch (3) is large and the ambient internal waves are small but larger than the patch with large amplitude and tilt angle, the patch and the strong density gradients at its boundary can be significantly tilted (3), middle panel Fig. 2. Vorticity is produced by baroclinic torques proportional to the cross product of the density gradient and the vertical pressure gradient vectors $\partial \vec{\omega} / \partial t \approx \nabla \rho \times \nabla p / \rho^{2}$.

Turbulence forms at the tilted boundaries of the patch, increasing its volume (4) and producing the larger, more spheroidal form shown middle right (5) in Fig. 2. The trajectory of an evolving patch on the log-log plot follows a $+1 / 3$ slope line as turbulence fossilizes and as zombie turbulence grows, as shown on a hydrodynamic phase 
diagram (HPD) at the bottom of Fig. 2. The Froude number ratio for a microstructure patch is $\mathrm{Fr} / \mathrm{Fr}_{0}=\left(\varepsilon / \varepsilon_{0}\right)^{1 / 3}$. The Reynolds number ratio for a microstructure patch is $\mathrm{Re} / \mathrm{Re}_{\mathrm{F}}$ $=\varepsilon / \varepsilon_{\mathrm{F}}$, Gibson (1986). The power of a patch is measured by an extrapolation to the original $\mathrm{Re}_{0} / \mathrm{Re}_{\mathrm{F}}$ value along its $+1 / 3$ trajectory.

An important semi-empirical result is that the $\mathrm{Re}_{0} / \mathrm{Re}_{\mathrm{F}}$ values of patches produced at the sea surface by breaking fossil and zombie turbulence waves approximates the $\mathrm{Re}_{0} / \mathrm{Re}_{\mathrm{F}}$ values of the fossils and zombies that radiate the waves, GBKL (2006). The mechanism and evidence from the ocean interior are described in Gibson (1987). Thus, $\varepsilon_{0}$ at the surface required by the BZTMA model is $\sim \mathcal{E}_{\mathrm{F}}$ $\mathrm{Re}_{0} / \mathrm{Re}_{\mathrm{F}}$, so we can estimate the surface power required for surface smoothing by turbulence from the surface stratification, surface mixing depth, and the $\mathrm{Re}_{0} / \mathrm{Re}_{\mathrm{F}}$ values of the dominant fossils indicated by HPD plots from sampled patches.

The zombie turbulence patch formation process is nonlinear and favors the largest fossil turbulence patches since these have the largest area for absorption of internal wave radiation and will grow more rapidly than the smaller ambient patches. Therefore, the zombie turbulence patches that dominate zombie wave radiation and the remote detection of submerged turbulence remnants will be large and rare, and correspondingly difficult to sample. Furthermore, the BBL fossil turbulence waves are nonlinear functions of the bottom boundary layer speed and direction, and are likely to be radiated in intermittent packets. Since both the zombies and the waves that power them are nonlinear, the zombie fossil waves will be beamed in vertical mixing chimneys that may or may not reach the surface to break and affect the brightness Fourier anomalies. Anomaly detections thus require large averaging areas to include adequate numbers of BZTMA mixing chimneys that reach the sea surface.

Figure 3 is a satellite image showing herringbone clouds. The clouds reveal narrow frequency band fossil turbulence waves emanating from the stratified turbulent boundary layer on the sea surface below. Contrary to the Richardson poem, turbulence always starts at small scales and cascades to larger scales until the energy supply is terminated or the growth is limited by walls or forces larger that the turbulence inertial vortex forces, Gibson (1991a).

Buoyancy forces of a stratified turbulent boundary layer terminate the vertical growth at the Ozmidov scale at beginning of fossilization $L_{R_{0}}$. After fossilization, fossil turbulence waves are radiated near vertically at a narrow frequency band with wavelength proportional to $L_{R_{0}}$. Such waves are a key factor in our BZTMA model. Notice that the herringbone clouds show a rather variable radiation of fossil turbulence waves in packets. Stripes and eddy patterns on the sea surface suggest turbulence formation at fronts produce fossil turbulence wave radiation to the surface and a remote detection of submerged turbulence similar to that of the present study. An extensive collection of laboratory, atmospheric, and numerical illustrations of such narrow frequency band internal waves and their effects are available in Bruce Sutherland's website (http://taylor.math.ualberta.ca/ bruce/).

\section{SATELLITE IMAGES AND BRIGHTNESS ANOMALY PATTERNS}

Figure 4 shows the surface brightness anomaly patterns computed by the Russian company ISINTECH from an Ikonos-2 satellite image taken September 2, 2003, Bondur (2004). A tropical storm produced cloudiness and sea states that prevented microstructure measurements. The strongest anomaly fragment was found to the NW (northwest) of the end of the diffuser with an EW (east-west) wavelength of $38 \mathrm{~m}$ and a NE (north-east) wavelength of $58 \mathrm{~m}$, shown by double arrows. A similar anomaly fragment (\#83) was detected by ISINTECH just NW of the end of the diffuser for the Sept. 2, 2002, RASP experiment, GBKL (Fig. 3).

Vertical profiles and the ship drift showed the wastewater convecting in this direction, so the BZTMA mechanism accounts for the observations. The source of vertical internal waves activating the smaller fossil density turbulent patches ( $6 \mathrm{~m} \mathrm{~L}_{\mathrm{Tmax}}$ ) to form zombie turbulence wave patterns with $58 \mathrm{~m} \mathrm{NE}, 38 \mathrm{~m} \mathrm{EW}$, and $75 \mathrm{~m} \mathrm{~N}$, wavelengths and directions are likely stratified bottom boundary layer turbulent events.

A more extensive and qualitatively different pattern of surface brightness anomaly fragments was found on Sept. 13, 2003, Bondur (2004), as shown in Fig. 5. Only one large lobe extending $20 \mathrm{~km}$ to the SW was observed, versus $10 \mathrm{~km} \mathrm{SW}$ and $7 \mathrm{~km}$ SE lobes as observed in RASP 2002, Sept. 2, and for Sept. 2, 2003, Fig. 4, and Sept. 14, 2003, Fig. 6. The area of the lobe is more than $200 \mathrm{~km}^{2}$, and goes beyond the optical satellite image (see ENVISAT radar image Fig. 17 bottom left).

A complex assortment of anomalous narrow bandwidth Fourier components was found, reflecting 90, 160, and $200 \mathrm{~m}$ wavelengths for one of the strong fragments as shown by double arrows, and oriented in a complicated directional pattern of unknown origin. The two strongest $2 \times 2 \mathrm{~km}$ fragments have slightly different Fourier components, all with large 100-200 m wavelengths. The strong anomalies are farther from the diffuser, more than $10 \mathrm{~km}$, so the fossil and zombie turbulence patches persisting to this distance will be in view of packets of fossil turbulence waves from bottom boundary layer turbulence in a greater variety of directions.

The tendency of BBL turbulence to radiate fossil turbulence waves in packets is a result of its nonlinear nature, with its power proportional to the bottom velocity cubed. The large size of the Sept. 13 lobe is attributed to strong offshore advection of Sand Island outfall fossils by estuarine pumping due to Sept. 10 rains. The location of the mixing front detected by the towed body is shown by the dotted line, Fig. 14.

Figure 6 shows the RASP 2002 two-lobed pattern of surface brightness anomalies, from a QuickBird satellite image on Sept. 14, 2003, Bondur (2004). Strong anomaly fragments are about 3 and $9 \mathrm{~km}$ to the $\mathrm{SW}$ and show 
Carl H. Gibson et al. / JAFM , Vol. 1, No. 1, pp. 11-42, 2008.

Fourier components in the wavelength range 100-200 m.

A prominent oil slick was observed emanating from an anchored ship. Red ovals reflect maxima in rms temperature gradients found by contouring vertical microstructure profiles on a section $7.5 \mathrm{~km}$ south of the diffuser, Fig. 8. These are interpreted as temperature fossils of intermittent BZTMA mixing chimneys. The blue arrow is the tow body path along the same section taken about an hour later, from Fig. 15. The red section of the arrow is a detected $900 \mathrm{~m}$ packet of $100 \mathrm{~m}$ wavelength internal waves.

\section{VERTICAL MICROSTRUCTURE PROFILES}

Figure 7 shows a Sept. 13, 2003 contour plot of the rms temperature dissipation rate from a sequence of 28 vertical profiles taken with the MSS dropsonde system from the ship HAPA at a distance of $5.5 \mathrm{~km}$ south of the outfall diffuser. A single, strong temperature-mixing region was found at the sea surface (green oval) that is interpreted as the fossil temperature turbulence remnant of a BZTMA mixing chimney. This illustrates the intermittent, patchy nature of the BZTMA process, and requires averaging over large surface fragments to capture the Fourier brightness anomalies.

Figure 8 is a contour map of rms temperature gradients for vertical profiles taken along a section $7.5 \mathrm{~km}$ south of the outfall diffuser on Sept. 14, 2003. Two regions of enhanced surface mixing are identified (red ovals). These are interpreted as fossil turbulence remnants of BZTMA mixing chimneys at the sea surface that contain the internal wavelength information used by ISINTECH to extract surface brightness anomalies.

Figure 9 shows GPS drogue tracks for the RASP 2003 experiments. Alongshore tidal currents advect fossils of the Sand Island outfall turbulence EW, except on Sept. 11 when there was a strong offshore estuarine flow driven by rains of Sept. 10 and BZTMA vertical mixing.

Figure 10 shows vertical Thorpe overturning scales near the diffuser, and a profile to the bottom at $85 \mathrm{~m}$ a kilometer to the East. Near the diffuser the surface Thorpe overturns reveal breaking of near vertically radiated fossil and zombie turbulence waves. The turbulent boundary layer radiates 20 $\mathrm{m}$ wavelength fossil turbulence waves to the surface at the Ozmidov scale at fossilization of the turbulence. But such large-scale near-vertical internal waves would be reflected rather than breaking and would therefore show no surface mixing effect. The BZTMA mechanism works because its smaller scale zombie turbulence waves can break at the base of the wind mixed layer and the fossils produced can smooth the surface capillary waves in detectable patterns.

Figure 11 shows sample vertical profiles for the vertical dropsonde section taken $5.5 \mathrm{~km}$ south of the outfall diffuser on Sept. 13, 2003. Profiles to the east of the mixing front show large Thorpe overturn scales, reflecting the Mixed Region of Fig. 17 (bottom). The BZTMA mechanism fails in the mixing region because the vertical stratification needed to transmit zombie turbulence waves near-vertically is too weak. Thorpe overturns in the green oval region show $10 \mathrm{~m}$ values significantly larger than the $6 \mathrm{~m}$ sizes of the largest outfall patches, suggesting that these patches may have extracted energy from ambient vertical internal waves to become zombie turbulence patches.

Figure 12 shows sample vertical profiles for the dropsonde section taken $7.5 \mathrm{~km}$ south of the outfall on Sept. 14, 2003. Thorpe overturn scales from the temperature dissipation rate maxima (red ovals) indicate BZTMA vertical mixing chimney channels formed from detected zombie outfall patches with scales up to $8 \mathrm{~m}$. The profile to the east shows only weak zombie waves will penetrate the well-mixed layer extending to $60 \mathrm{~m}$, even though a zombie patch with $20 \mathrm{~m}$ vertical scales is detected.

\section{HORIZONTAL MICROSTRUCTURE PROFILES}

Figure 13 shows an MSS mounted with padding in the catamaran towed sensor platform (CATFISH), attended by Hartmut Prandke and Pak Tao Leung. The platform decouples vertical motions of the ship by slack tethers yoked above and below to the forward wing with the towrope through the wing-pulley to the depressor. CATFISH weighs $10 \mathrm{~kg}$ for easy handling on a small ship. High molecular weight polymer ropes are stronger than stainless steel and minimize vibrational effects on the sensors.

Information from the sensors is transmitted to the ship laboratory for recording through the data cable, which is loosely attached to the $6 \mathrm{~mm}$ towrope during deployment. Speeds up to $3 \mathrm{~m} \mathrm{~s}^{-1}$ were used, but higher speeds and multiple platforms may be needed in future experiments to properly characterize the highly intermittent and patchy vertical mixing processes that permit remote detections of submerged turbulence. Data analysis and interpretation of the large volume of horizontal towed microstructure profiling information is at an early stage.

Figure 14 shows the tow body detection in the temperature, density, and temperature gradient signal of the mixing front to the east of the large single lobe anomaly region of Sept. 13, 2003, Figs. 5 and 7 . Tows were $2 \mathrm{~m} \mathrm{~s}^{-1}$ at $12 \pm 0.1 \mathrm{~m}$ depths, $5.5 \mathrm{~km}$ south of the outfall. To the west of the mixing front in the anomaly lobe sufficient stable stratification exists to permit the BZTMA transmission of information about the existence of submerged fossil outfall density turbulence patches. Temperature and density fluctuations and temperature and density gradients are small. To the east of the mixing front the temperature and density fluctuations and gradients abruptly increase, reflecting the large overturning scales of Fig. 11 caused by the BZTMA estuarine mixing of Sept. 11, Fig. 17.

Figure 15 shows a $900 \mathrm{~m}$ packet of $100 \mathrm{~m}$ wavelength $\approx 3$ $\mathrm{m}$ amplitude internal waves in the westernmost maximum rms temperature gradient region, from the Sept. 14, 2003 section $7.5 \mathrm{~km}$ south of the outfall diffuser, shown by the western red oval in Fig. 8 and Fig. 11. The packet coincides with the medium intensity Fourier brightness anomaly of fragment 47 of Fig. 6, as shown in that figure. 


\section{HYDRODYNAMIC PHASE DIAGRAMS}

Figure 16 shows all the HPD microstructure points calculated for RASP 2002 and 2003 experiments. No actively turbulent patches with $\mathrm{Re}_{0} / \mathrm{Re}_{F} \approx 25,000$ were detected in the samples from RASP 2002 (top) as expected by extrapolation along the $+1 / 3$ slope decay locus. Thus, the outfall turbulence has been under-sampled. From the RASP 2003 HPD plot (bottom) it was inferred from the 3518 patches that the $\mathrm{Re}_{0} / \mathrm{Re}_{\mathrm{F}}$ ratio for the Sand Island outfall in 2003 was also 25,000, extrapolating to the three actively turbulent patches detected, large circles. Using this for RASP 2002 it appears that the 2130 microstructure patches collected have under sampled the process since no actively turbulent patches were detected with $\mathrm{Re} / \mathrm{Re}_{\mathrm{F}}>$ 8,000. The Gibson (1986) HPD method was employed, Leung and Gibson (2004).

Actively turbulent patches must have supercritical Froude and supercritical Reynolds numbers. In Fig. 16, $\mathrm{Fr} / \mathrm{Fr}_{0}=$ $\left(\varepsilon / \varepsilon_{0}\right)^{1 / 3}$ for a patch is computed from the measured $\varepsilon$ value normalized by the value at the beginning of fossilization $\varepsilon_{0}$, where $\varepsilon_{0}$ is $3 \mathrm{~L}_{\mathrm{Tmax}}{ }^{2} \mathrm{~N}^{3}$. The Reynolds number ratio $\varepsilon / \varepsilon_{\mathrm{F}}$ is computed using the viscous dissipation rate at complete fossilization $\varepsilon_{\mathrm{F}}=30 v \mathrm{~N}^{2}$. Microstructure patches were identified using the Prandke and Stips (1992) and Gregg (1980) method of zero crossings, where $>20 \mathrm{~cm}$ of no zero vertical temperature gradient is required between vertically separated patches.

The collection of 5521 microstructure HPD points in Mamala Bay shown in Fig. 16 is large enough to permit a variety of conclusions about stratified ocean turbulence. Clearly there is no convergence to the Dillon (1982, 1984) correlation, where turbulence is created continuously with $\varepsilon$ proportional to $\varepsilon_{0}$ and with no fossil turbulence. Both Dillon conclusions are incorrect. Sufficient active-fossil patches were collected that the original completely-active outfall turbulence patches were also included, even a few with large values of $\mathrm{Re}_{0} / \mathrm{Re}_{\mathrm{F}}$. Very few fully fossil turbulence patches were found in the lower left quadrant, which can be attributed to the zombie turbulence process in which fossil turbulence patches extract energy from ambient fluid motions.

A band of patches for the RASP 2002 and RASP 2003 HPD plots of Fig. 16 are labeled zombie turbulence patches, and extrapolate along $+1 / 3$ slope loci to $\operatorname{Re}_{0} / \operatorname{Re}_{\mathrm{F}}$ values $\sim 100,000$. We find no reason to believe these are fossils of such powerful events. Instead, these points represent patches of fossil density turbulence that have extracted ambient gravitational potential energy from internal wave and other motions to produce zombie turbulence. This increases their $\varepsilon_{0}$ values by increasing their vertical overturn scales $L_{T \max }$, with a resulting increase in their $+1 / 3$ extrapolated $\operatorname{Re}_{0} / \operatorname{Re}_{\mathrm{F}}$ values.

Significantly more zombie turbulence patches are found in the Fourier brightness anomaly regions of Figs. 4-6 than in ambient regions, as shown in the following section (Fig. 18). For a given $\mathrm{Re}_{0} / \mathrm{Re}_{\mathrm{F}}$ value, many more active-fossil HPD patches are found than active turbulence HPD patches. As $\operatorname{Re}_{0} / \mathrm{Re}_{\mathrm{F}}$ increases the percentage of active patches decreases. This reflects the tendency for intermittency to increase with Reynolds number for turbulence (Kolmogorov's third similarity law), and the difficulty of adequate microstructure sampling in the deep ocean where a very few powerful turbulence events typically dominate the entire range of mixing and diffusion processes.

Estimates of average vertical diffusivities and average dissipation rates were made during the HOME experiments without taking the possibility of fossil turbulence or intermittency effects into account, Alford et al. (2006), Klymak et al. (2006), Lee et al. (2006). No HPDs were presented, and no intermittency parameters. Such averages may be over-estimates but are more likely under-estimates.

\section{DISCUSSION OF RESULTS}

Figure 17 shows RADARSAT and ENVISAT radar images of the outfall region for Sept. 11, 2003, (a. top), and Sept. 13, 2003, (a. bottom), analyzed for surface brightness anomalies by a variety of ISINTECH algorithms, Bondur (2004). Radar images are adjusted for increased contrast using methods including speckle noise filtration by Median filters and Gaussian equalization. Slight changes in surface smoothing at capillary wave scales can result in anomalies in the sea surface brightness histograms from Bragg resonance. These are compared to microstructure sea truth results for temperature and viscous dissipation rate contours at the locations shown for the two days.

Temperature dissipation rate (b. top) and viscous dissipation rate (b. bottom) contours are shown for dropsonde microstructure sections of these quantities. Radar brightness anomalies detected as are interpreted as caused by BZTMA mixing chimney smoothing triggered by Sand Island outfall fossils advected alongshore by tidal motions and offshore by estuarine salt wedge mixing. Strong BZTMA vertical mixing in Mamala Bay by Sand Island outfall fossil turbulence patches was triggered by Sept. 10 rains on Oahu Island, producing amplified estuarine offshore flows and a mixing region shown at the top of Fig. 17 that obliterated the possibility of remotely detecting submerged turbulence from the outfall on Sept. 11, 2003. Zombie turbulence waves in the mixed region of Sept. 11 south of the outfall were so weakened that they could not smooth the sea surface and be detected, but were quite effective in the NS red region (top left Fig. 17) of estuarine mixing extending nearly $40 \mathrm{~km}$ to the SSE. Alongshore BZTMA mixing triggered by the Sand Island fossil turbulence patches is shown extending $30 \mathrm{~km}$ east.

By Sept. 13, 2003, the EW locations of the mixed and mixing regions reversed (Fig. 17, bottom), giving the large one lobe surface brightness anomaly pattern observed from the Ikonos-2 optical image, Figs. 5, 7, 14 and Fig. 17 bottom.

Figure 18 shows the HPD points for RASP 2003 sorted according to whether the patches were in or out of the brightness anomaly regions, Wolk et al. (2004). Fig. 18 a. includes the diffuser itself in order to detect the original active and fossil turbulence outfall patches, shown as red 
Carl H. Gibson et al. / JAFM , Vol. 1, No. 1, pp. 11-42, 2008.

triangles. These fall on the $+1 / 3$ locus and indicate an initial Reynolds number ratio at beginning of fossilization $\mathrm{Re}_{0} / \mathrm{Re}_{\mathrm{F}}$ of 25,000 compared to only $\mathrm{Re}_{0} / \mathrm{Re}_{\mathrm{F}}$ of 300 for ambient patches inferred from Fig. 18c.

Even though 3518 patches were analyzed, the outfall turbulence process is still somewhat under-sampled, with only three patches in the fully turbulent quadrant of the HPD. No active patches were detected with $\mathrm{Re}_{0} / \mathrm{Re}_{\mathrm{F}}$ values larger than 25,000. Thus, the patches with large extrapolated $\mathrm{Re}_{0} / \mathrm{Re}_{\mathrm{F}}$ values are interpreted as zombie turbulence patches formed from fossil outfall turbulence patches by the BZTMA mechanism. Few zombie patches were found in the ambient plot Fig. 18c. These appear to be weak zombies with insufficient forcing by ambient vertical internal wave motions to form BZTMA mixing chimneys.

The arithmetic mean dissipation rate for patches of Fig. 18a was found to be $8.1 \times 10^{-7} \mathrm{~m}^{2} \mathrm{~s}^{-3}$, larger by a statistically significant factor of 5 (with 95\% confidence) than in the ambient regions of Figs. 18b and c, Wolk et al. (2004). This enhanced viscous dissipation rate gives $5 \mathrm{kw}$ of outfall pumping power in the $6 \times 10^{6} \mathrm{~m}^{3}$ region of the diffuser, but when spread over the $>10^{3}$ larger volumes of $>100 \mathrm{~km}^{2}$ ISINTECH detected brightness anomaly regions it requires $>5$ megawatts.

This reflects a dramatic turbulent power amplification by the BZTMA mechanism, where fossil outfall turbulence patches extract large quantities of ambient internal wave power as their zombies radiate information to the sea surface about the submerged fossil outfall turbulence remnants and the bottom boundary layer fossil Ozmidov turbulence scales $L_{R_{0}}$ of the vertical internal waves that activate the zombies.

\section{CONCLUSIONS}

The RASP 2003 microstructure experiments confirm and extend the ability of Russian colleagues to remotely detect remnants of submerged turbulence as demonstrated in RASP 2002, Bondur and Filatov (2003), KBG, GBKL. The beamed zombie turbulence maser action (BZTMA) mechanism, Fig. 1d, continues to be the most viable working hypothesis for the physical processes leading to remote detection capabilities of both optical and radar satellites, GBKL.

Examination of energetics supports the BZTMA model, where fossil density turbulence patches advected from the outfall extract energy and information from powerful bottom boundary layer generated FTW packets of narrow frequency band internal waves and beam it to the sea surface at smaller scales as ZTWs that can interfere with wind roughening but in the larger scale bottom FTW patterns that are detectable as Fourier brightness anomalies.

BZTMA mixing from the Hawaiian ridge may also convert astronomically generated barotropic tidal waves to baroclinic internal tidal waves, Fig. 1a, and produce remotely-detectable soliton-like waves from river fronts, Fig. 1b. A significant fraction of the turbulent kinetic energy at fossilization is radiated near-vertically as FTWs and ZTWs.

Basic turbulence cascade mechanisms driven by inertial vortex forces $\vec{v} \times \vec{\omega}$ from small scales to large and the vertical stratified turbulence mixing process by fossil turbulence maser action are reviewed, Fig. 1c. All evidence shows that oceanographic studies of vertical transports of heat, mass, momentum and energy are affected by these fundamental turbulence and fossil turbulence internal wave processes at all scales and at every stage.

Oceanographic budgets of conserved quantities will never balance if turbulence is assumed to occur at the last stage of a cascade of internal waves from large scales to small, and if fossil turbulence is assumed to be nonexistent or irrelevant rather than a central dynamical component of vertical oceanic transport processes.

Dropsonde and towed body microstructure evidence suggests the BZTMA process is a powerful vertical mixing mechanism. The nonlinearity of zombie turbulence formation, where the largest zombie patches have the largest cross section for extracting turbulence energy from ambient internal waves, leads to a focus of the process into small vertical regions termed BZTMA mixing chimneys.

These mixing chimneys triggered by the Sand Island outfall fossils appear to dominate the vertical and horizontal mixing processes of Mamala Bay. Estuarine mixing is produced by BZTMA vertical momentum transport, where the volume flow rate of fresh water runoff from the island is amplified by vertical mixing with seawater.

The resulting deep offshore flows advect fossil turbulence patches from the Sand Island outfall trapping depth to produce the observed SW and SE brightness anomaly lobes under average rain conditions, and the large SW brightness anomaly lobe of Sept. 13, 2003, following strong rain conditions of Sept. 10.

Nearly six thousand HPD points were collected in RASP 2002 and 2003. These strongly support the fossil turbulence paradigm for stratified turbulence, where actively turbulent patches are formed at small scales and cascade to larger scales until the maximum Thorpe overturning scale $\mathrm{L}_{\text {Tmax }}$ is $\sim 0.6$ times the Ozmidov scale $L_{R_{0}}=\left(\varepsilon_{0} / N^{3}\right)^{1 / 2}$ at fossilization and the stratification frequency $N$ is computed for vertical density differences somewhat larger than $\mathrm{L}_{\text {Tmax }}$.

The normalized Reynolds number for fully active outfall turbulence patches appears to be about $\operatorname{Re}_{0} / \operatorname{Re}_{\mathrm{F}}=\varepsilon_{0} / \varepsilon_{\mathrm{F}}=$ 25,000 , the viscous dissipation rate at complete fossilization $\varepsilon_{\mathrm{F}}=30 \mathrm{vN}^{2}$ and $v$ is the viscous dissipation rate. Patches that extrapolate to larger $\mathrm{Re}_{0} / \mathrm{Re}_{\mathrm{F}}$ values are interpreted as zombie turbulence patches. The lack of observed patches in the fully fossilized turbulence quadrant is evidence that the zombie turbulence vertical mixing and diffusion process is generic to stratified turbulent mixing and diffusion in the ocean. 
Carl H. Gibson et al. / JAFM , Vol. 1, No. 1, pp. 11-42, 2008.

Since only a few patches were detected with $\mathrm{Re} / \mathrm{Re}_{\mathrm{F}}=\varepsilon / \varepsilon_{\mathrm{F}}$ $\geq 25,000$, it is concluded that these fully active outfall turbulence patches have brief lifetimes compared to those in the active-fossil state and rapidly enter the active-fossil HPD quadrant as $\varepsilon$ decays but the overturn scale does not. From the large detection areas and measured advection speeds the fossil outfall turbulence patches and their zombies persist for time periods exceeding $1000 \mathrm{~N}^{-1}$.

Measured average viscous dissipation rates in the anomaly regions are significantly higher than those measured in ambient regions, Wolk et al. (2004), demonstrating the remarkable amplification of kilowatts of pumping power input at the outfall into the megawatt range by the BZTMA mechanism, drawing on gigawatts of available bottom boundary layer fossil turbulence waves and possibly other sources of ambient internal wave energy.

Future measurements are needed to confirm and better quantify these interpretations. Important geometrical questions remain about the BZTMA mixing chimneys. Are they chimneys or sheets? What fraction of horizontal sea surfaces do the chimneys occupy as a function of depth? How do they depend on time and ambient oceanic conditions? Oceanographic and limnological mixing and diffusion processes may be significantly affected by the BZTMA mechanism.

\section{ACKNOWLEDGEMENTS}

Fabian Wolk of Rockland Oceanographic Services Inc., and Hartmut Prandke of ISW Wassermesstechnik, supplied the MSS equipment, helped plan and carry out the measurements, and made numerous contributions to the validation, analysis and interpretation of the data. Financial support was provided by Directed Technologies, Inc.

\section{REFERENCES}

Alford, M.H., Gregg, M.C. \& Merrifield, M.A. (2006). Structure, propagation, and mixing of energetic baroclinic tides in Mamala Bay, Oahu, Hawaii, J. Phys. Oceanogr., 36, 997-1018.

Baker, M.A. \& Gibson, C.H. (1987). Sampling Turbulence in the Stratified Ocean: Statistical Consequences of Strong Intermittency, J. Phys. Oceanogr., 17(10), 18171837.

Bondur, V.G. \& Savin, A.I. (1995). Principle of modeling of signal fields at input into remote sensing equipment of aerospace environment monitoring systems, Research of the Earth From Space, 4, 24-33.

Bondur V.G. \& Filatov N.N. (2003). Study of physical processes: coastal zone for detecting anthropogenic impact by means of remote sensing. Proc. of the 7th Workshop on Physical Processes in Natural Waters, 2 5 July, 2003, Russia, Petrozavodsk, 98-103.

Bondur, V. G., Ed., (2004). ISINTECH Preliminary
Report, Remote Monitoring of the Anthropogenic Influence on Ecosystems in Littoral Waters (RASP), Phase III Remote sensing of Anthropogenic Influence caused by submerged sewers on ecosystems of Littoral Waters using optical and radiophysical space methods, January, 1-290.

Bondur, V.G. (2005). Presentation at RASP 2005 UCSD workshop, Mar. 9-11.

Cernicharo, J., Pardo, J.R. and Weiss, A. (2006). A new water vapor megamaser, The Astrophysical Journal, 646:L49-L52.

Dillon, T.R. (1982). Vertical overturns: A comparison of Thorpe and Ozmidov scales. J. Geophys. Res., 87, 9601-9613.

Dillon, T.R. (1984). The energetics of overturning structures: Implications for the theory of fossil turbulence, J. Phys. Oceanogr., 14, 541-549.

Driscoll, C.F., Jin, D.Z., Schecter, D.A. \& Dubin, D.H.E. (2002). Vortex dynamics in 2D electron plasmas, Physica C, 369, 588.

Egbert, G.D. \& Ray. R.D. (2003). Semi-diurnal and diurnal tidal dissipation from TOPEX/Poseidon altimetry, Geophys. Res. Lett., 30, 17, 1907, doi:10.1029/2003GL017676.

Fisher, H.B., List, E.J., Koh, R.C.Y., Imberger J. \& Brooks. N.H. (1997). Mixing in inland and coastal waters. Academic Press Inc., 483 p.

Garrett, C. (2003). Mixing with latitude, Nature, 422, 477-478.

Gibson, C.H. (1980). Fossil temperature, salinity, and vorticity turbulence in the ocean. In: Marine Turbulence, J.C.J. Nihoul, Jr, Editor, Elsevier Publishing Co., New York, 221-257.

Gibson, C.H. (1986). Internal waves, fossil turbulence, and composite ocean microstructure spectra, J. Fluid Mech., 168, 89-117.

Gibson, C.H. (1987). Oceanic turbulence: big bangs and continuous creation, PhysicoChemical Hydrodynamics, 8, 1-22.

Gibson, C.H. (1991a). Kolmogorov similarity hypotheses for scalar fields: sampling Intermittent turbulent mixing in the ocean and galaxy, in Turbulence and stochastic processes: Kolmogorov's ideas 50 years on, Proceedings of the Royal Society London, Ser. A, 434, 1890, 149-164; http://xxx.lanl.gov, astro-ph/9904269. 
Carl H. Gibson et al. / JAFM , Vol. 1, No. 1, pp. 11-42, 2008.

Gibson, C.H. (1991b). Turbulence, mixing, and heat flux in the ocean main thermocline, J. Geophys. Res., 96(C7), 20,403-20,420.

Gibson, C.H., Bondur, V.G., Keeler, R.N. \& Leung, P.T. (2006). Remote sensing of submerged oceanic turbulence and fossil turbulence, Int. J. Dyn. Fluids, in press: GBKL.

Gregg, M.C. (1980). Zero crossings of temperature microstructure. In: Marine Turbulence, J.C.J. Nihoul, $J r$, Editor, Elsevier, New York, 135-142.

Gregg, M.C., Sanford, T.B., \& Winkel, D.P. (2003). Reduced mixing of the breaking of internal waves in equatorial waters, Nature, 422, 513-515.

Keeler, R. N., Bondur, V.G. \& Gibson, C.H. (2005). Optical satellite imagery detection of internal wave effects from a submerged turbulent outfall in the stratified ocean, Geophys. Res. Lett., 32, L12610, doi:10.1029/2005GL022390: KBG.

Keeler, R.N., Bondur, V.G. \& Vithanage, D. (2004). Sea truth measurements for remote sensing of littoral water, Sea Technology 45(4), 53.

Klymak, J. et al. (2006). An estimate of tidal energy lost to turbulence at the Hawaiian Ridge, J. Phys. Oceanogr., 36, 1148-1164.

Koechner, W. (1999). Solid-State Laser Engineering, Fifth Revised and Updated Edition, Springer, NY.

Lee, C.M. et al. (2006). Internal tides and turbulence along the 3000-m isobath of the Hawaiian Ridge, J. Phys. Oceanogr., 36, 1165-1181.

Leung, P.T. \& Gibson, C.H. (2004). Turbulence and fossil turbulence in oceans and lakes, Chinese $J$. of Oceanology and Limnology, 22(1), 1-23. http:// xxx.lanl.gov, astro-ph/ 0310101.

Levine, M.D. \& Boyd, T.J. (2006). Tidally-forced internal waves and overturns observed on a slope: Results from HOME, J. Phys. Oceanogr., 36, 1184-1201.

Linden, P.F. (1975). The deepening of a mixed layer in a stratified fluid, J. Fluid Mech., 71, 385-405.

Lozovatski, I., Morozov, E., \& Fernando, H. (2003). Spatial decay of energy density of tidal internal waves, J. Geopys. Res., 108, 3201, doi:10.1029/2001Jc001169.

Munier, P. \& Leweke, T. (2003). Analysis and treatment of errors due to high velocity gradients in particle image velocimetry, Experiments in Fluids 35, 408-421.

Munk, W. \& Wunsch, C. (1998). Abyssal Recipes II: Energetics of tidal and wind mixing, Deep-Sea Res., 45, 1977-2010.
Nash, J.D. \& Moum J.N. (2005). River plumes as a source of large-amplitude internal waves in the coastal ocean, Nature, 437, 400-403.

Petrelis, F., Smith, S.L. \& Young, W.R. (2006). Tidal conversion at a submarine ridge, J. Phys. Oceanog. 36(6), 1053-1071.

Prandke, H. \& Stips, A. (1992). A model of Baltic thermocline turbulence patches, deduced from experimental investigations, Continental Shelf Research, Vol. 12, No. 5/6, pp. 643-659.

Pinkel, R. \& Rudnick, D. (2006). Editorial, J. Phys. Oceanog., 36, 965-966.

Rainville, L. \& Pinkel, R. (2006). Propagation of lowmode internal waves through the ocean, J. Phys. Oceanog., 36, 1220-1236.

Rudnick, D.L., Boyd, T.J., Brainard, R.E, Carter, G.S., Egbert, G.D., Gregg, M.C., Holloway, P.E., Klymak, J.M., Kunze, E., Lee, C.M., Levin, M.D., Luther, D.S., Martin, J.P., Merrifield, M.A., Moum, J.N., Nash, J.D., Pinkel, R., Rainville, L., Sanford, T.B. (2003). From tides to mixing along the Hawaiian Ridge, Science, 301(8), 355-357.

Sutherland, B.R. (2001). Finite-amplitude internal wavepacket dispersion and breaking, J. Fluid Mech. 429, 343-380.

Wolk, F., Prandke, H., \& Gibson, C.H. (2004). Turbulence measurements support satellite observations, Sea Technology, 48(8), 43.

Wolk, F., Prandke, H. \& Leung, P.T. (2004). RASP 2002/2003 Data Analysis, Preliminary Results and Measurement Plan. 1-13. 
Carl H. Gibson et al. / JAFM , Vol. 1, No. 1, pp. 11-42, 2008.

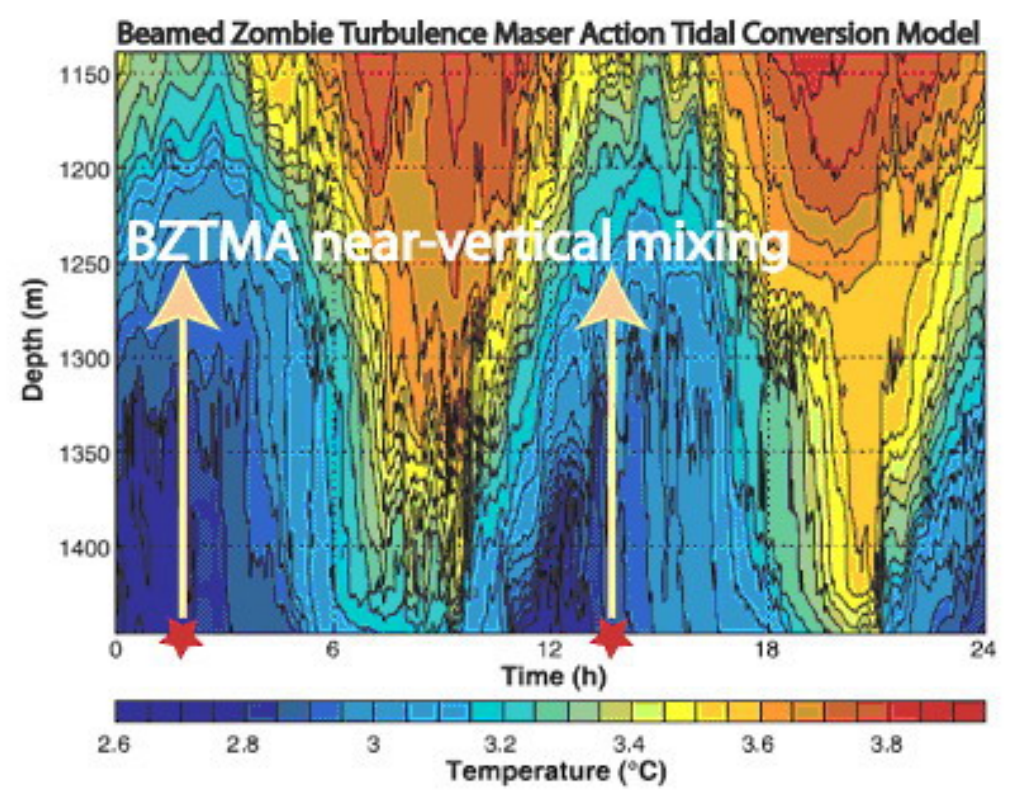

Fig. 2. Temperature as a function of depth and time, measured on a mooring on the flank of the ridge in water with a depth of $1453 \mathrm{~m}$ during spring tide. The position of the mooring is shown by the yellow cross in Fig. 1. At the semidiurnal period, the peakto-peak displacements are $300 \mathrm{~m}$.

Rudnick et al. 2003

Fig. 1a - How is the energy of barotropic tides converted to baroclinic internal tides on the Hawaiian ridge? Rudnick et al. 2003 (the HOME collaboration) assume the energy cascade is directly from barotropic to baroclinic at the slope, and that the internal tide is somehow responsible for the increased turbulence and temperature dissipation rates, as observed over the ridge by Levine and Boyd (2006). An alternative hypothesis is that the $300 \mathrm{~m}$ amplitude $0.7{ }^{\circ} \mathrm{C}$ vertical temperature differences are produced by enhanced near-vertical BZTMA mixing from semi-diurnal period tidal bottom turbulence on topography. The BZTMA tidally mixed regions then propagate away from the ridge with approximate speed $c \approx \sqrt{g \rho^{\prime} h}$ as internal tides, where $\rho^{\prime}=\Delta \rho / \rho$ and $h$ is the vertical amplitude of the mixed region. Increased dissipation rates observed are due to breaking FTWs and ZTWs of the BZTMA mechanism, not breaking of the internal tide. 
Carl H. Gibson et al. / JAFM , Vol. 1, No. 1, pp. 11-42, 2008.
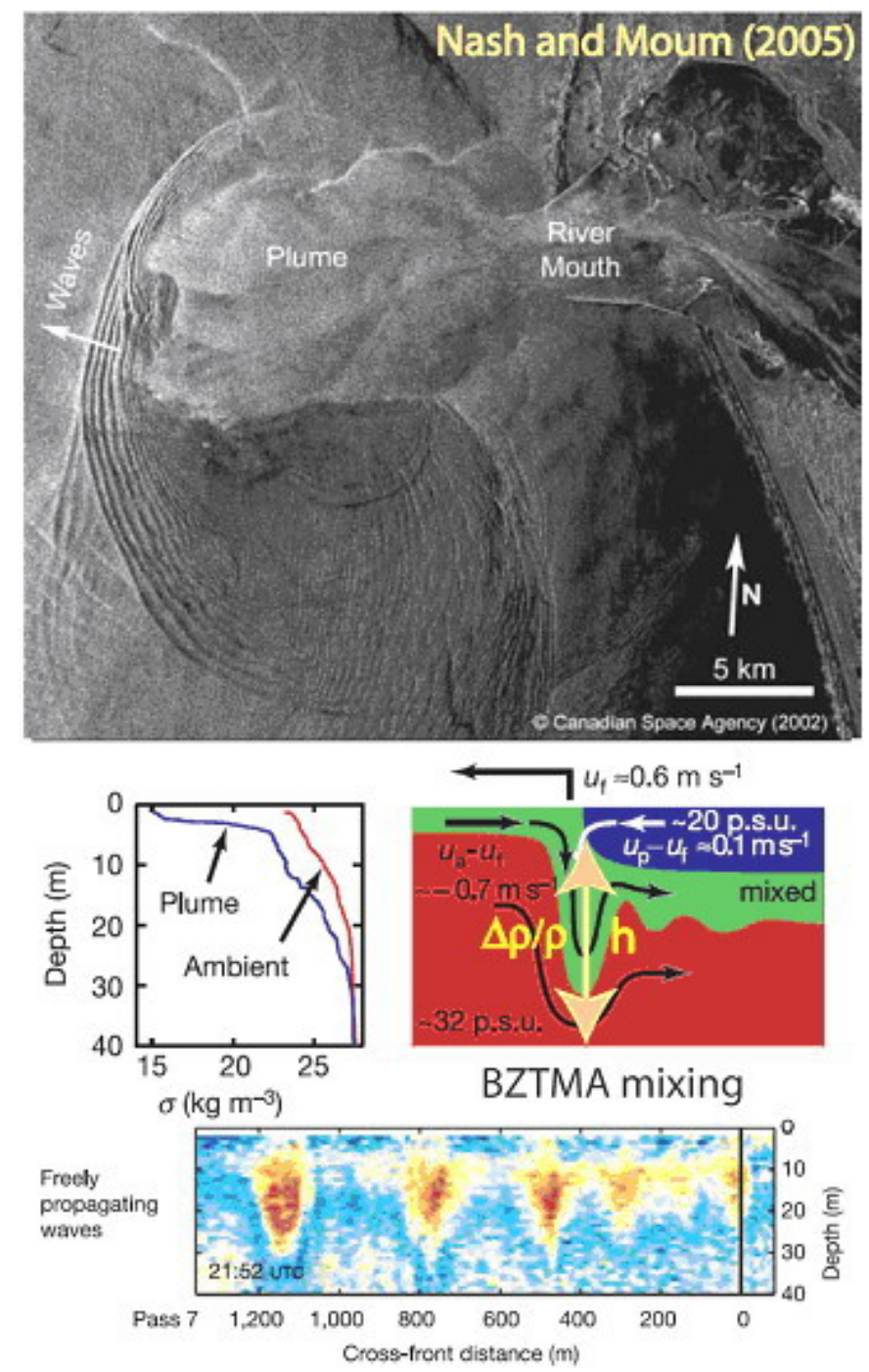

Fig. 1b - Formation of internal tidal waves at the Columbia River plume front, Nash and Moum (2005). The amplitude and speed $c \approx \sqrt{g \rho^{\prime} h}$ of the waves is determined by the density difference $\rho^{\prime}=\Delta \rho / \rho$ and penetration depth $h$ of BZTMA mixing zone. Surface manifestations of the waves detected by the synthetic aperture radar (SAR) image at the top are transmitted near-vertically by small scale FTWs from turbulence produced by the solitons. 


\section{a. The turbulence cascade is from small scales to large}

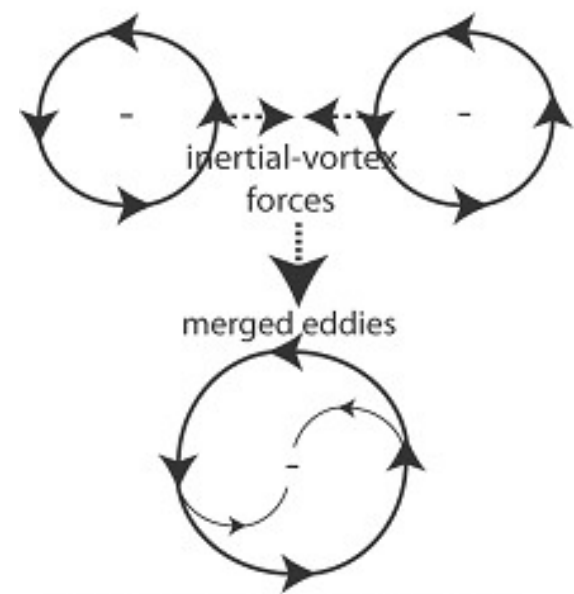

eddies with the same spin merge

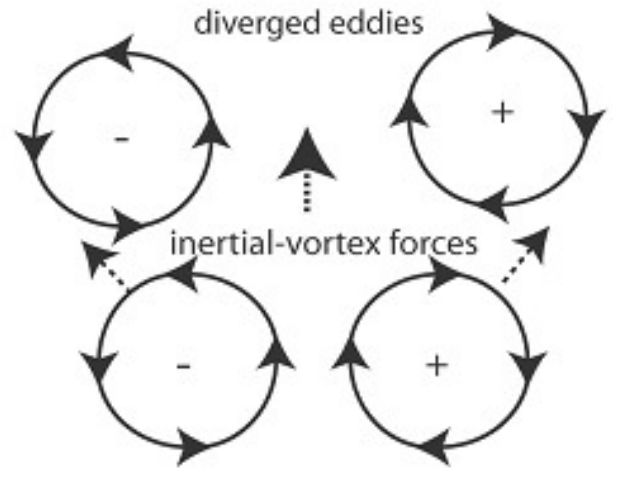

eddies with opposite spin diverge

b. Turbulence powers vertical internal wave maser action
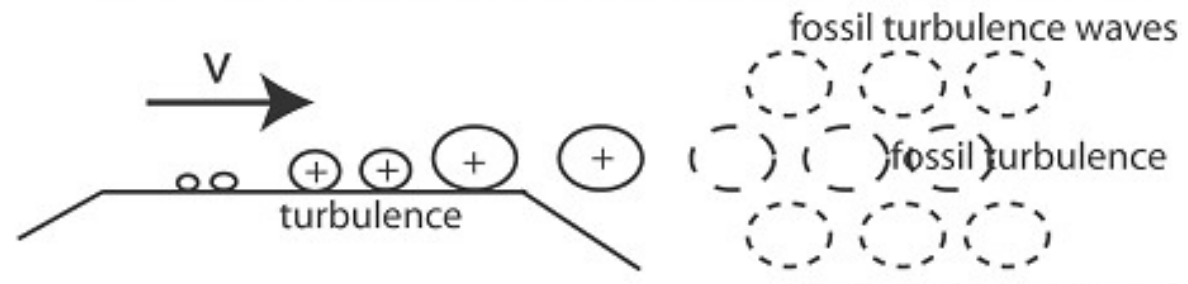

fossil turbulence waves

Fig. 1c - Physical mechanisms of turbulence and stratified turbulence. a. Vortex mechanisms of the turbulence cascade from small scales to large. Adjacent eddies with the same vorticity produce inertial vortex forces $\vec{v} \times \vec{\omega}$ (dashed arrows) that cause merging. Nearby eddies with opposite spin diverge and expand the turbulent region driven by $\vec{v} \times \vec{\omega}$ forces. b. Turbulence, fossil turbulence, and fossil-turbulence-waves in a stratified fluid produce internal-wave maser-action where turbulent kinetic energy fossilized by buoyancy forces is radiated as fossil turbulence waves (FTWs). Because the vertical velocity of the FTWs matches the horizontal velocity of the turbulence source, the angle of propagation is a near-vertical $45^{\circ}$. Because a substantial fraction of the turbulent kinetic energy is radiated near-vertically in narrow-wavenumber-band FTWs with $\lambda \approx L_{R_{0}}$ this is an efficient hydrodynamic maser-action. 
Carl H. Gibson et al. / JAFM , Vol. 1, No. 1, pp. 11-42, 2008.

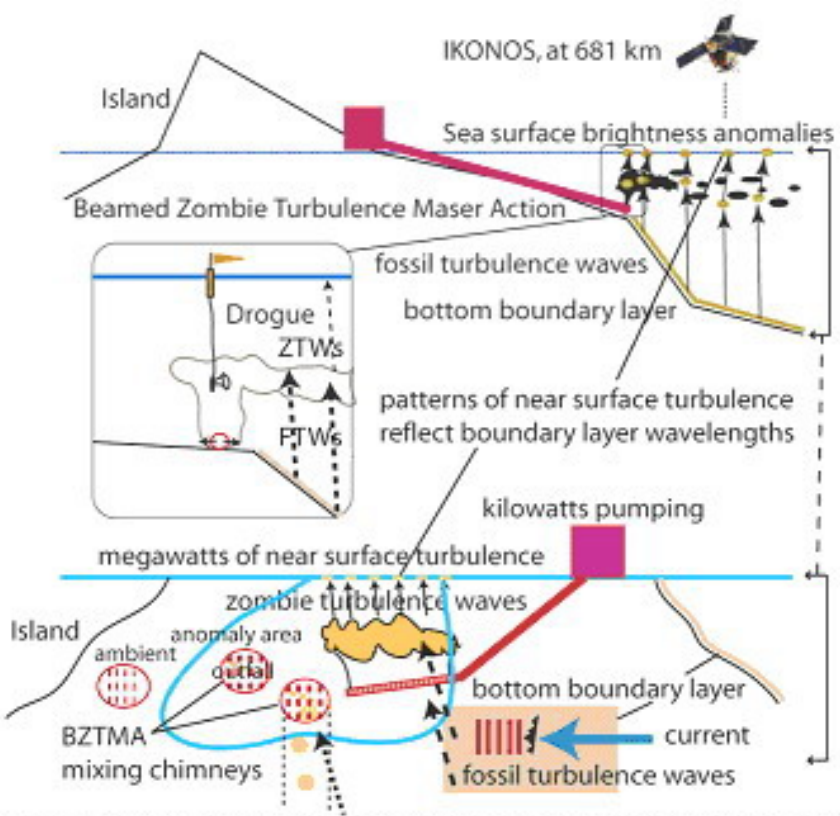

BZTMA: potentially gigawatt power levels are available from perfectly rough bottom boundary layer turbulence to produce FTWs that radiate near vertically and can be trapped on the pycnocline. These have wavelengths near the Ozmidov scale $L_{R 0}$ at beginning of fossilization. These solitons trigger $Z$ TW radiation to the surface.

Fig. 1d - Beamed zombie turbulence maser action (BZTMA) model for the RASP remote detection of submerged outfall turbulence and fossil turbulence. See text for discussion. 
Carl H. Gibson et al. / JAFM , Vol. 1, No. 1, pp. 11-42, 2008.

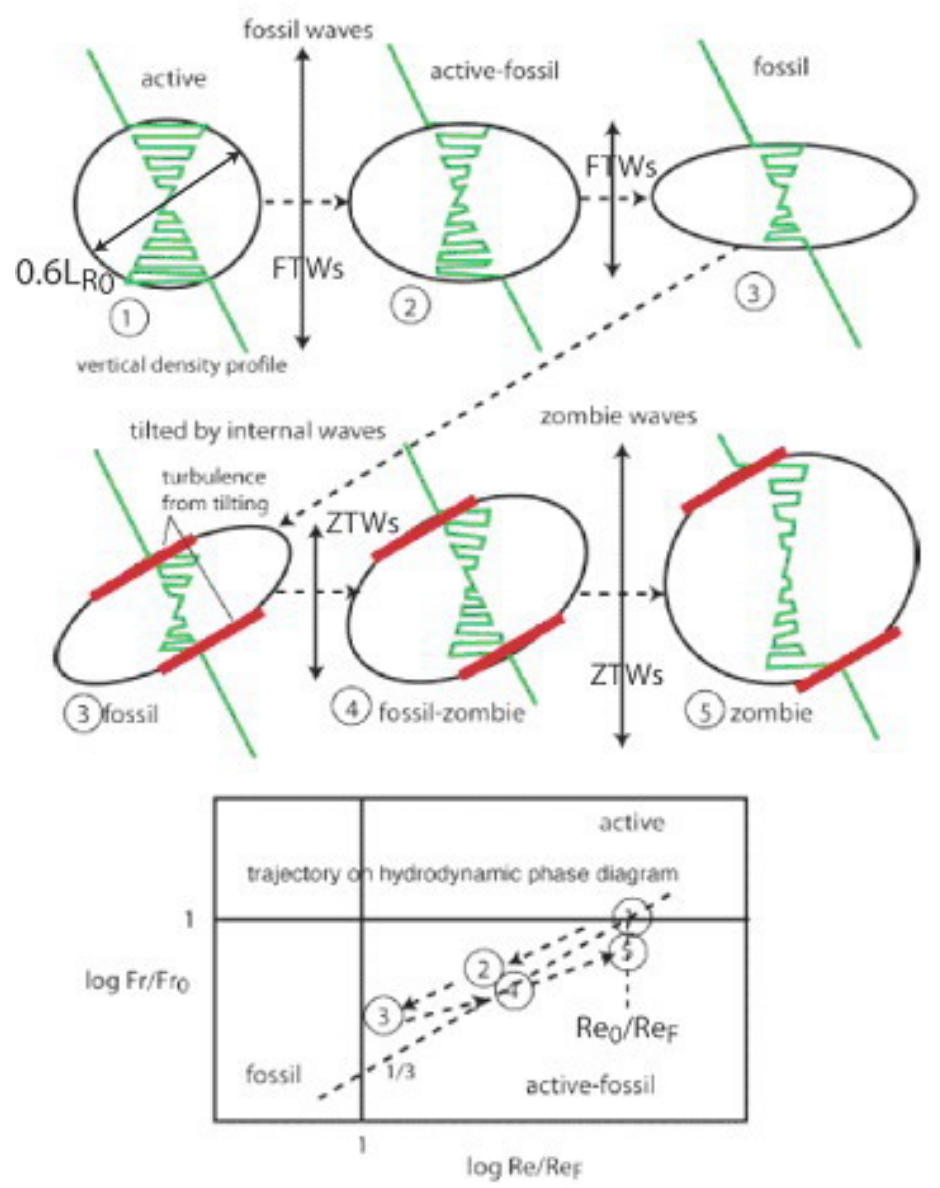

Fig. 2 - Evolution of an actively turbulent patch (1) in the stratified ocean. Stages are numbered (1)-(5). The vertical density mixes as an active-fossil patch (2) and begins to flatten after it radiates FTWs and approaches complete fossilization (3). The patch is tilted by ambient internal waves to form a zombie turbulence patch in (4) and (5). Energy extracted from the ambient internal wave by turbulence formation on the zombie boundaries is radiated as ZTWs. The trajectory of the patch stages is shown on the hydrodynamic phase diagram at the bottom. Patch (1) is at the beginning of fossilization with critical Froude number and $\mathrm{Re}_{0} / \mathrm{Re}_{\mathrm{F}}=$ $\varepsilon_{0} / \varepsilon_{\mathrm{F}}$. The patch evolves along the dashed line locus with slope $\sim 1 / 3$, moving down toward fossilization (1), (2), (3), and then back up as the growing zombie turbulence patch forms (3), (4), (5). 
Carl H. Gibson et al. / JAFM , Vol. 1, No. 1, pp. 11-42, 2008.

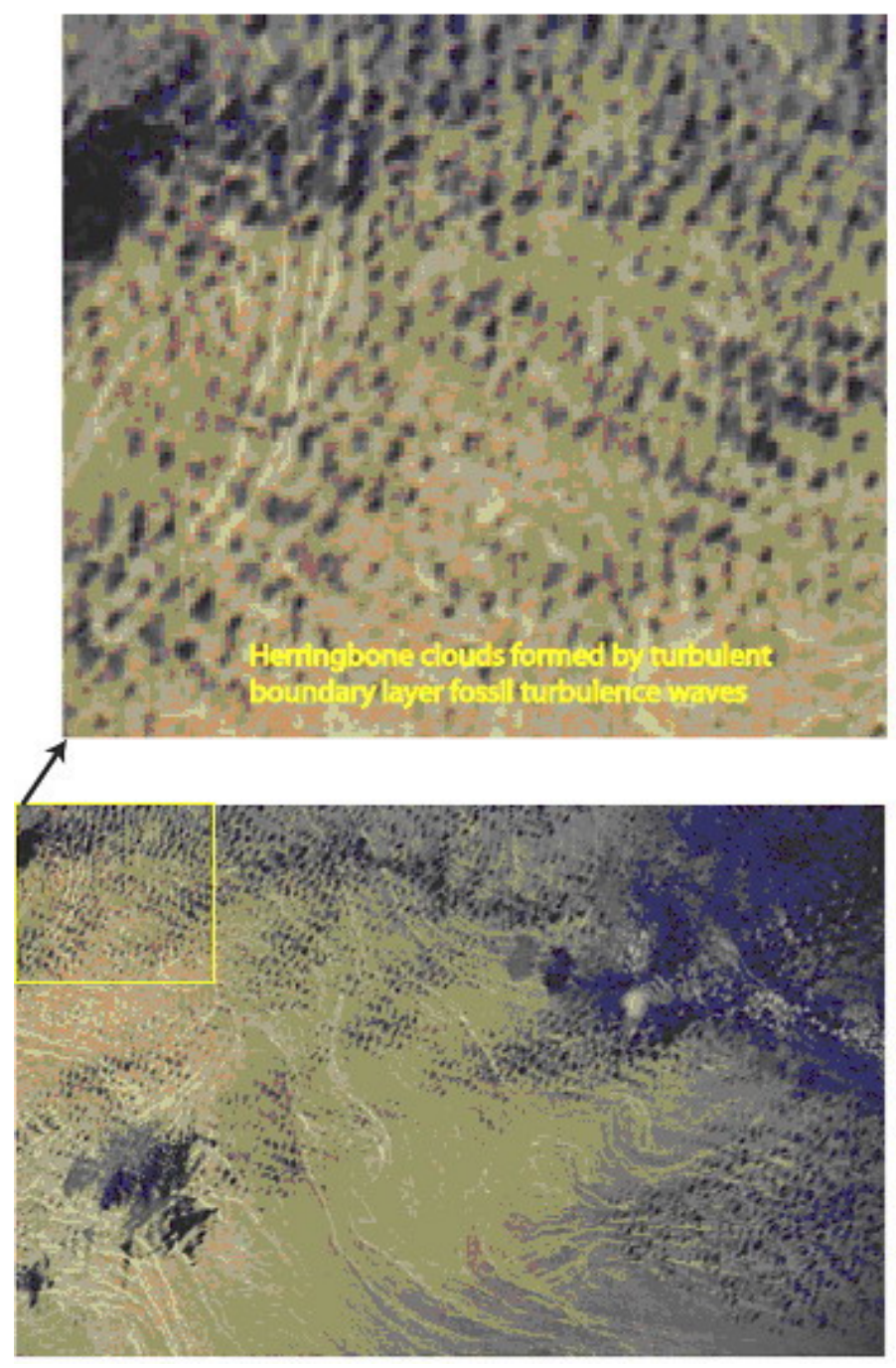

Fig. 3 - Herringbone clouds are formed near the surface of the sea from a wind generated turbulent boundary layer fossilized in a narrow frequency band at the Ozmidov scale of turbulence fossilization by the stably stratified atmosphere. Fossil turbulence waves (FTWs) radiate near-vertically to create the atmospheric Herringbone clouds. Note the ocean surface brightness patterns. The present study suggests these eddy signatures are caused by FTWs radiating (probably in BZTMA mixing chimneys) from submerged turbulence produced on eddy fronts. 
Carl H. Gibson et al. / JAFM , Vol. 1, No. 1, pp. 11-42, 2008.

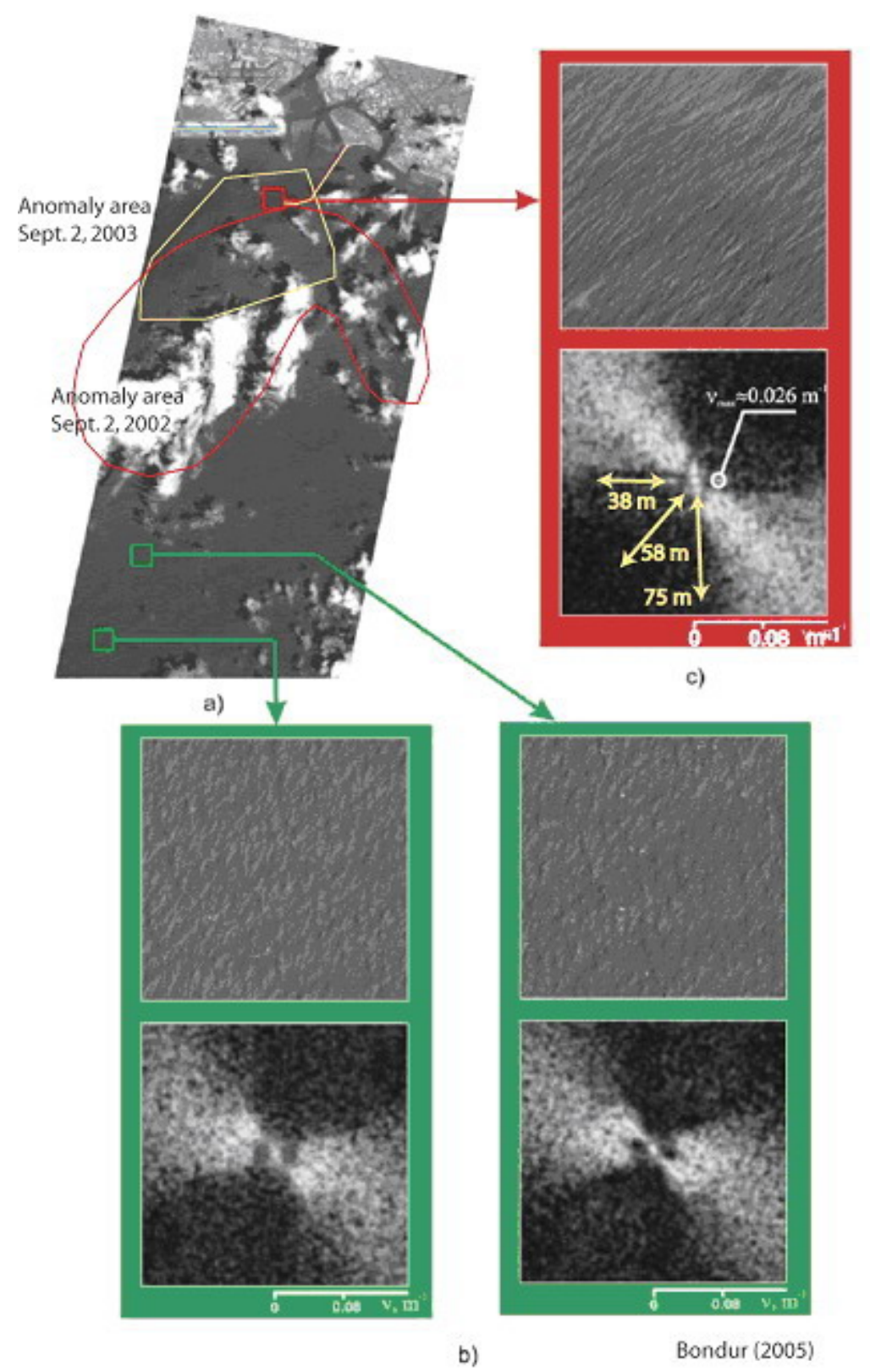

Fig. 4 - Surface brightness anomaly patterns from an Ikonos-2 optical image, Sept. 2, 2003, Bondur (2004). The strongest anomaly fragment (a) was found NW of the end of the diffuser pipe, with intense 38, 58 and $75 \mathrm{~m}$ wavelength Fourier components oriented EW, NE and N (double arrows) that are not present in background fragments (b). Anomaly areas for Sept. 2 observations in 2002 and 2003 are outlined. See Gibson et al. (2006) for the anomaly maps. 
Carl H. Gibson et al. / JAFM , Vol. 1, No. 1, pp. 11-42, 2008.

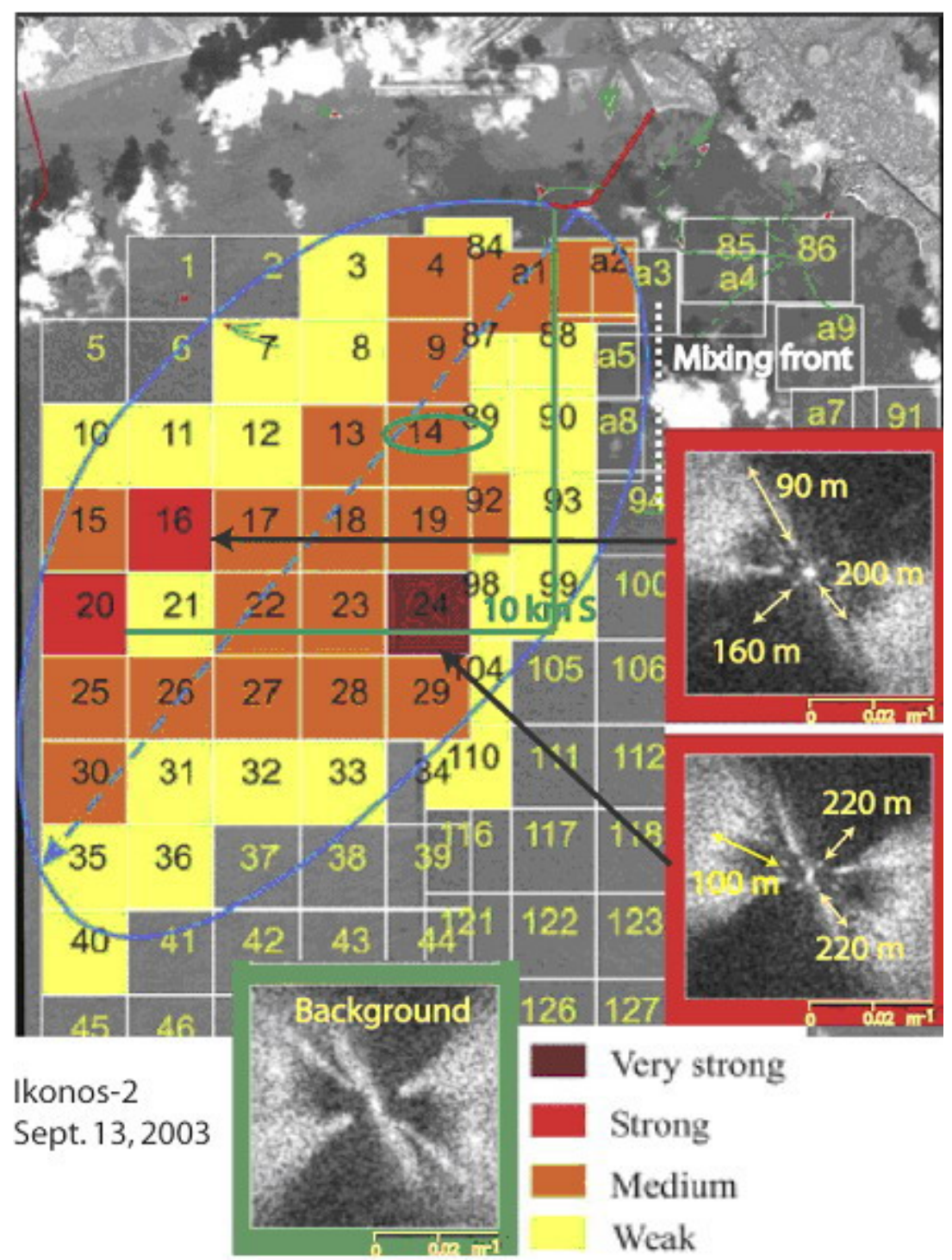

Fig. 5 - Surface brightness anomaly patterns from an Ikonos-2 optical image, Sept. 13, 2003, Bondur (2004). The strongest Fourier components are at wavelengths of 90, 160 and $200 \mathrm{~m}$, and are at distances of 8-10 km SW of the diffuser. Rather than two lobes extending $10 \mathrm{~km} \mathrm{SW}$ and $7 \mathrm{~km} \mathrm{SE}$, we see only one $20 \mathrm{~km} \mathrm{SW}$ lobe covering $200 \mathrm{~km}^{2}$ and extending outside the area of satellite image coverage. Anomaly fragments shown are $2 \mathrm{x} 2 \mathrm{~km}$ in size, but $1 \mathrm{x} 1 \mathrm{~km}$ fragments covered the same area. The green oval corresponds to the maximum in rms temperature gradients for the $5.5 \mathrm{~km}$ vertical profiling contours, Fig. 7. The mixing front location from towed measurements of Fig. 14 is shown by a dashed line. 
Carl H. Gibson et al. / JAFM , Vol. 1, No. 1, pp. 11-42, 2008.

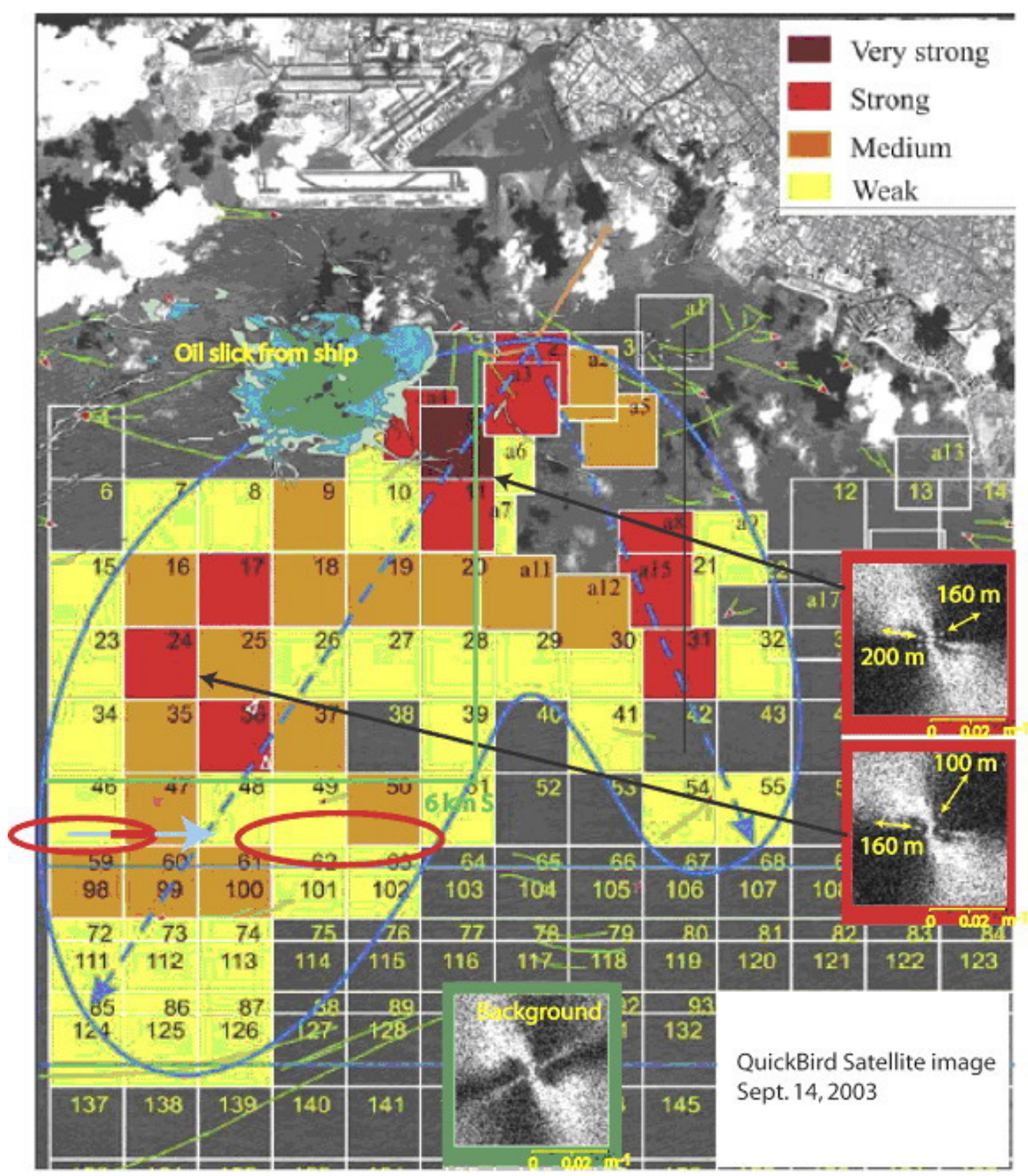

Fig. 6 - Surface brightness anomaly patterns from a QuickBird optical image, Sept. 14, 2003, Bondur (2004). The strongest Fourier components are at wavelengths of 100, 160 and $200 \mathrm{~m}$. Two anomaly lobes were detected extending $22 \mathrm{~km} \mathrm{SW}$ and $7 \mathrm{~km} \mathrm{SE} \mathrm{covering} 400 \mathrm{~km}^{2}$. The two red ovals in fragments 16, 17, 18, 19 correspond to near surface maxima of temperature dissipation rates observed in the vertical microstructure section, in Fig. 8. 
Carl H. Gibson et al. / JAFM , Vol. 1, No. 1, pp. 11-42, 2008.

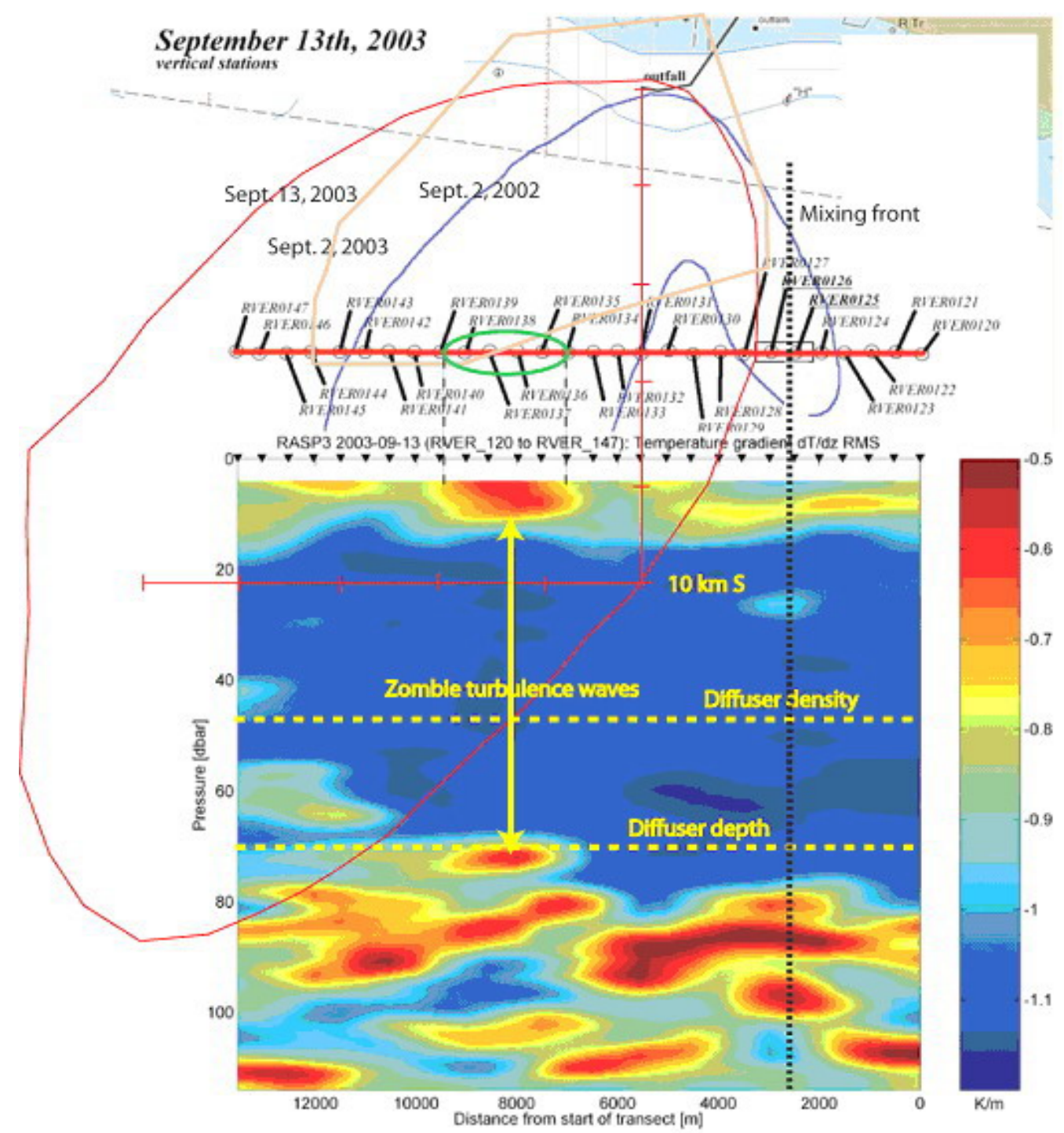

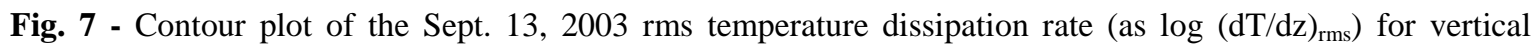
profiles $5.5 \mathrm{~km}$ south of the diffuser. Active near surface regions of this indicator of fossil temperature turbulence are interpreted as evidence of breaking zombie turbulence waves (double arrows) above the wastewater trapping depth. The Sept. 13, 2003 area of surface brightness anomalies is about $200 \mathrm{~km}^{2}, \sim 4$ times larger than that for Sept. 2, 2002. 
Carl H. Gibson et al. / JAFM , Vol. 1, No. 1, pp. 11-42, 2008.

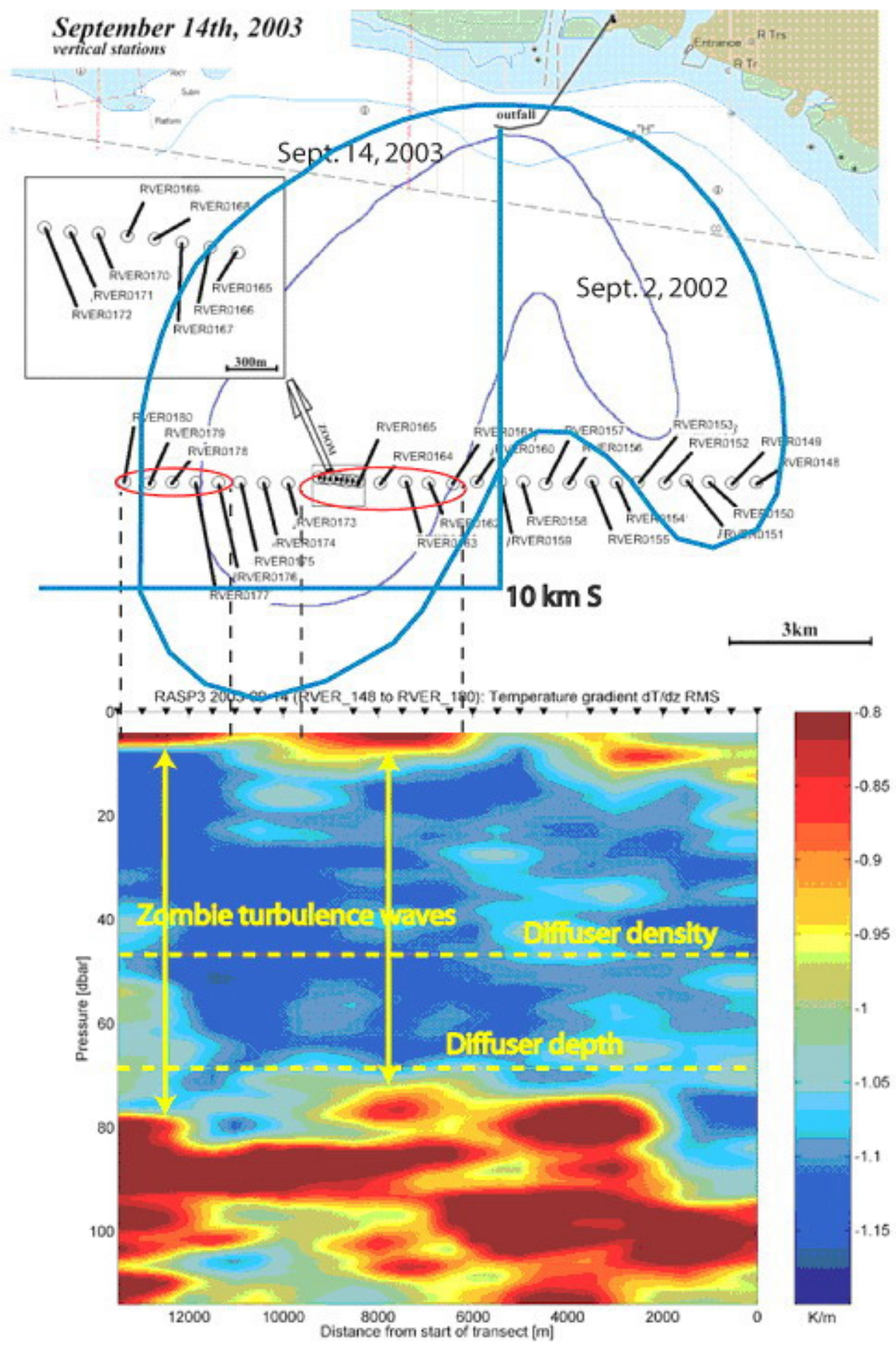

Fig. 8 - Contour plot of the Sept. 14, 2003 rms temperature dissipation rate for vertical profiles $7.5 \mathrm{~km}$ south of the diffuser. The Sept. 14, 2003 area of surface brightness anomalies is somewhat larger than that for Sept. 2, 2002. 
Carl H. Gibson et al. / JAFM , Vol. 1, No. 1, pp. 11-42, 2008.

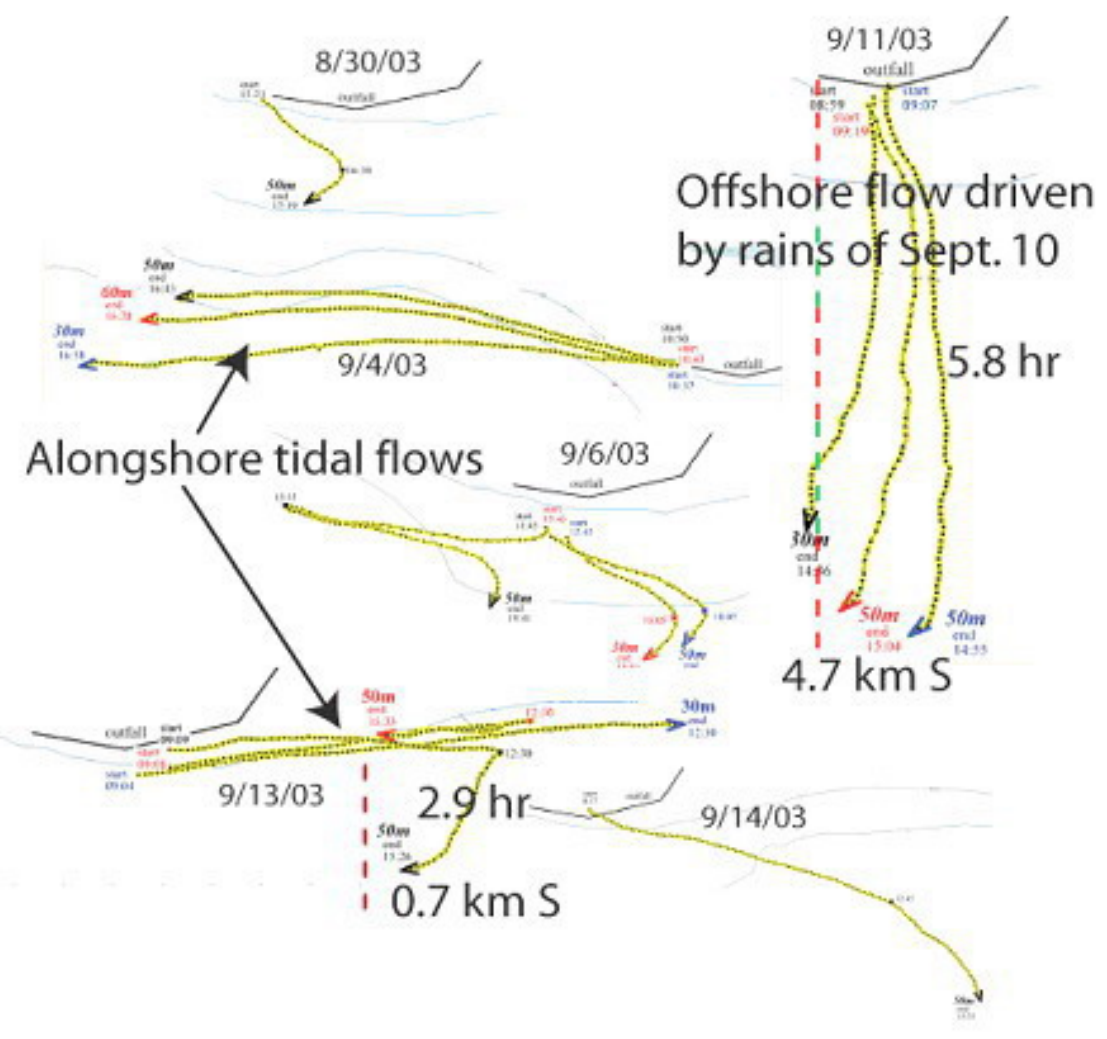

Fig. 9 -Parachute drogue tracks for RASP 2003. The drifters were inserted near the wastewater diffuser at 30$50 \mathrm{~m}$ near the trapping depth. GPS positions are shown as dots along the paths. EW tidal motions $\sim 0.2 \mathrm{~m} \mathrm{~s}^{-1}$ usually were larger than NS onshore and offshore drifts $\sim 0.03 \mathrm{~m} \mathrm{~s}^{-1}$. The exception is the strong offshore deep currents for Sept. 11, 2003, that is attributed to estuarine pumping from strong rains of Sept. 10, driven by BZTMA vertical mixing, Fig. 17. 


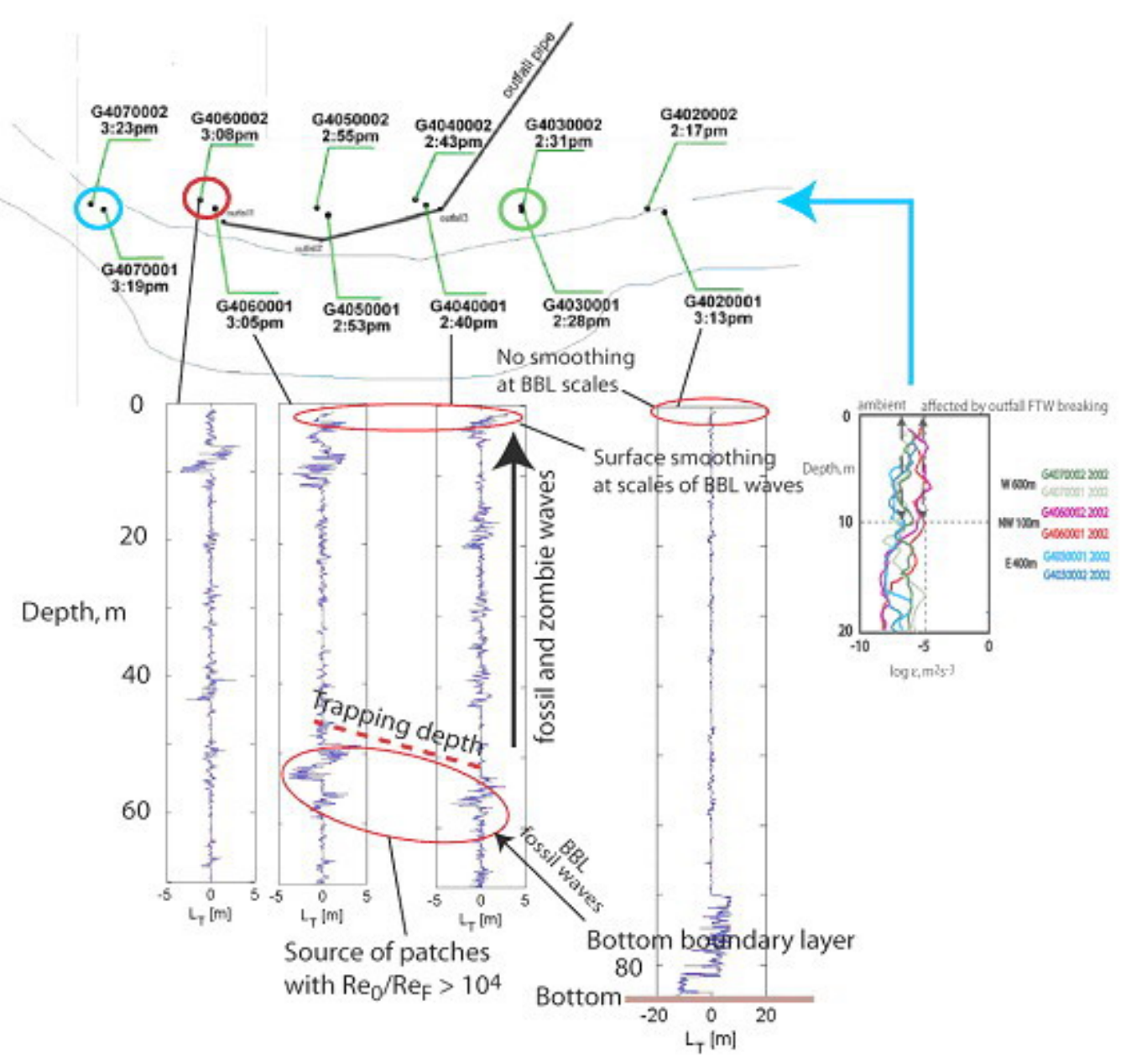

Fig. 10 - Vertical Thorpe overturn scale profiles $L_{T}$ near the diffuser, and one a kilometer to the east all the way to the bottom that reveals a $15 \mathrm{~m}$ thick turbulent boundary layer at $85 \mathrm{~m}$ depth. Notice that there is no $\mathrm{L}_{\mathrm{T}}$ expression at the sea surface of this boundary layer that could cause sea surface smoothing, as there is for stations near the outfall where smaller scale fossil and zombie turbulence waves are possible. Outfall $\varepsilon$ values in the upper $10 \mathrm{~m}$ are $10^{-5} \mathrm{~m}^{2} \mathrm{~s}^{-3}$ from FTW and ZTW breaking (red circle), two orders of magnitude larger than ambient values (green and blue circles). 
Carl H. Gibson et al. / JAFM , Vol. 1, No. 1, pp. 11-42, 2008.

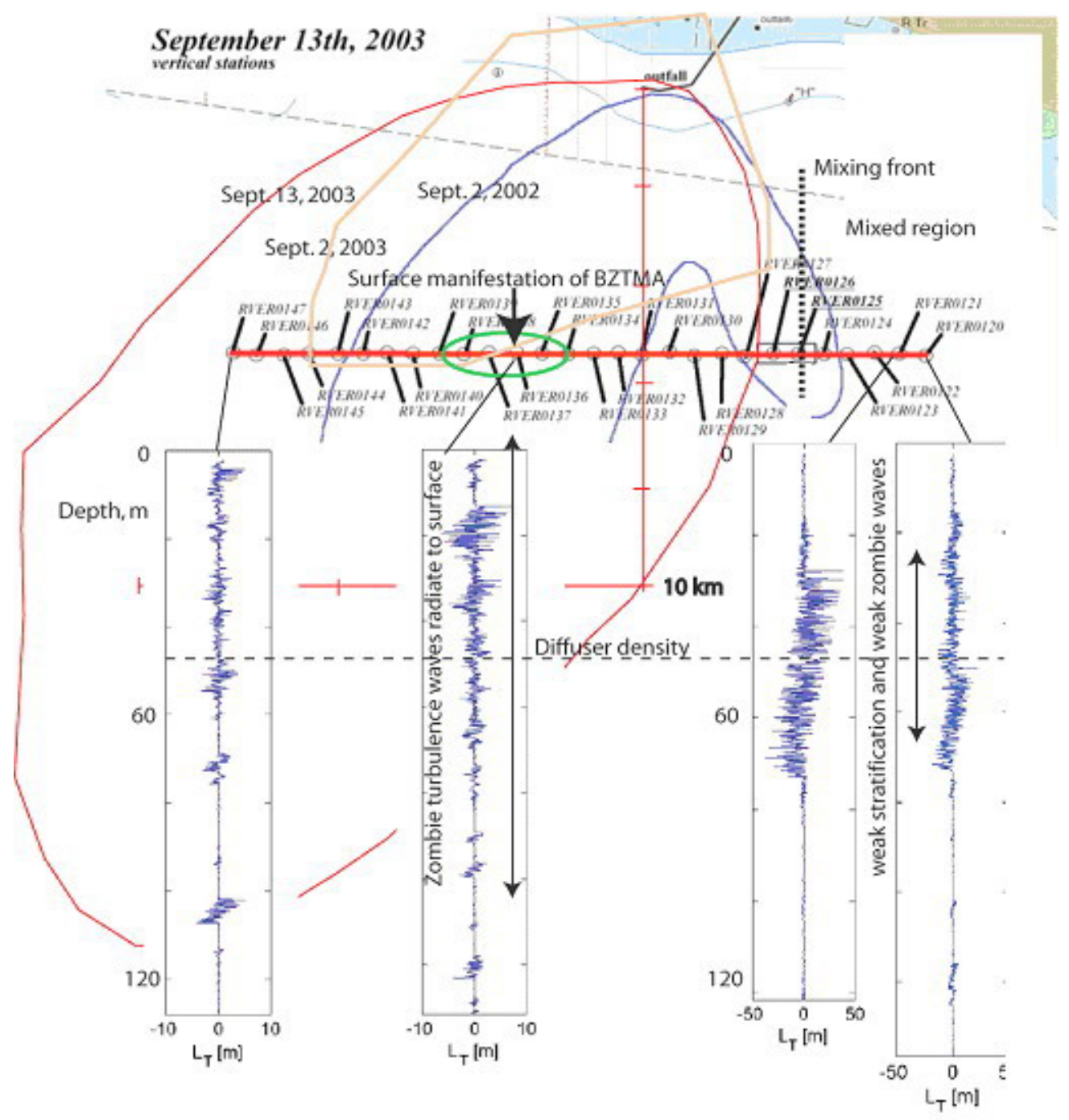

Fig. 11 - Vertical Thorpe overturn profiles at $5.5 \mathrm{~km}$ south of diffuser Sept. 13, 2004. Very large overturns $\mathrm{L}_{\mathrm{Tmax}}>30 \mathrm{~m}$ to the east show weak stratification permits the growth of large zombie turbulence patches with weak radiation to the surface. Smaller $8 \mathrm{~m}$ zombie turbulence overturns to the west are somewhat larger than the original $6 \mathrm{~m}$ wastewater fossils and can effectively beam information to the surface about their presence in patterns of the 100-200 m BBL fossil waves. 
Carl H. Gibson et al. / JAFM , Vol. 1, No. 1, pp. 11-42, 2008.

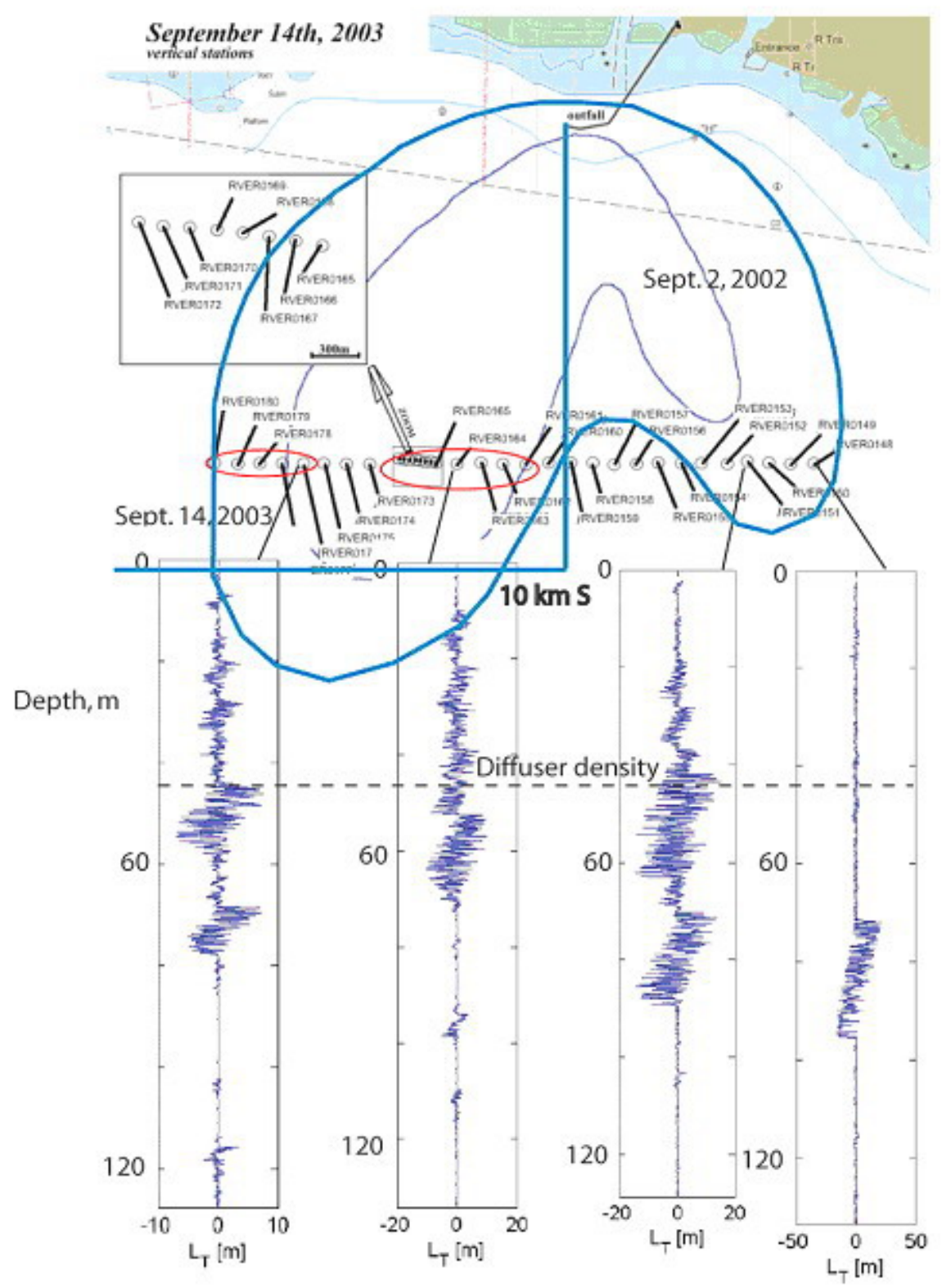

Fig. 12 - Vertical Thorpe overturn profiles at $7.5 \mathrm{~km}$ south of diffuser Sept. 14, 2004. Profiles in the two BZTMA mixing chimneys to the west shown by red ovals show 6-10 m maximum overturn scales advected offshore along the diffuser pycnocline. The two profiles to the east show zombie turbulence patches with 10-20 $\mathrm{m} \mathrm{L}_{\mathrm{Tmax}}$ values that have mixed away the vertical density gradient above, decreasing their detectability. 
Carl H. Gibson et al. / JAFM , Vol. 1, No. 1, pp. 11-42, 2008.

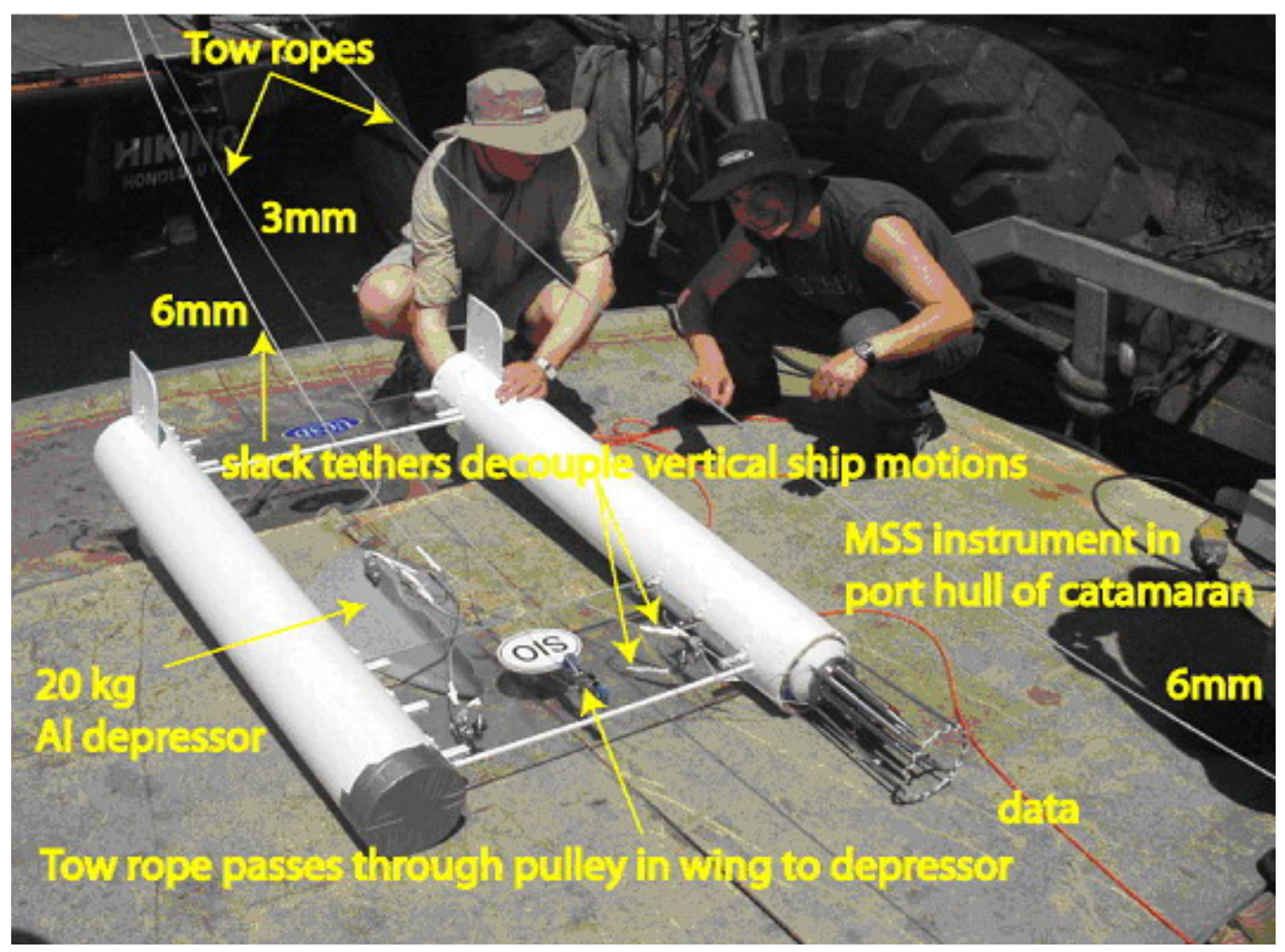

Fig. 13 - Catamaran tow body with MSS instrument mounted in port hull. Tow speeds of $2 \mathrm{~m} \mathrm{~s}^{-1}$ exceeded the maximum speed permitted for shear sensors. Micro-temperature, micro-conductivity, micro-turbidity sensors and mean $\mathrm{T}, \mathrm{C}$ and $\mathrm{P}$ sensors were operational. 
Carl H. Gibson et al. / JAFM , Vol. 1, No. 1, pp. 11-42, 2008.

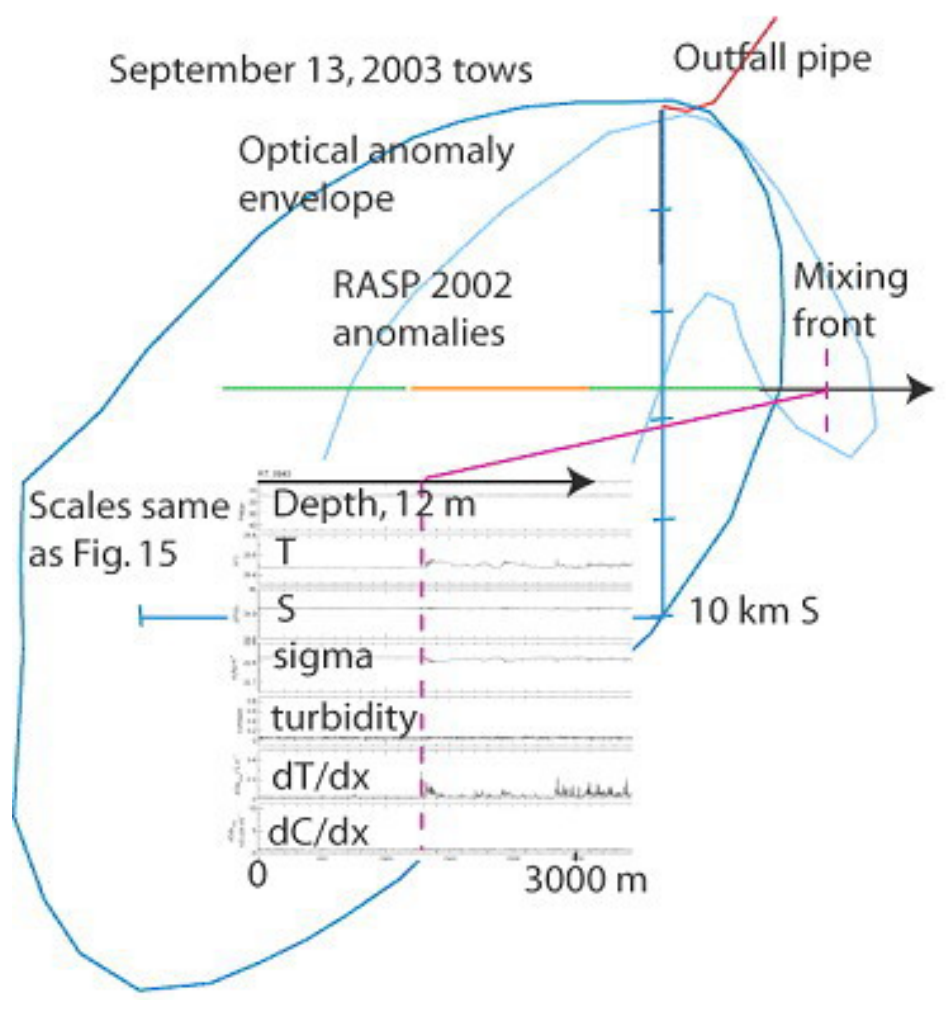

Fig. 14 - Horizontal profiles Sept. 13, 2003, compared to sea surface brightness anomaly patterns (Figs. 5 and 7). A mixing front was detected at the indicated position outside the anomaly area, confirming the vertical profiles of Fig. 11 that suggest the BZTMA near-vertical internal waves (ZTWs) in the SE lobe have mixed away so much of the stratification that no more information can be transmitted near-vertically (Fig. 17). 
Carl H. Gibson et al. / JAFM , Vol. 1, No. 1, pp. 11-42, 2008.

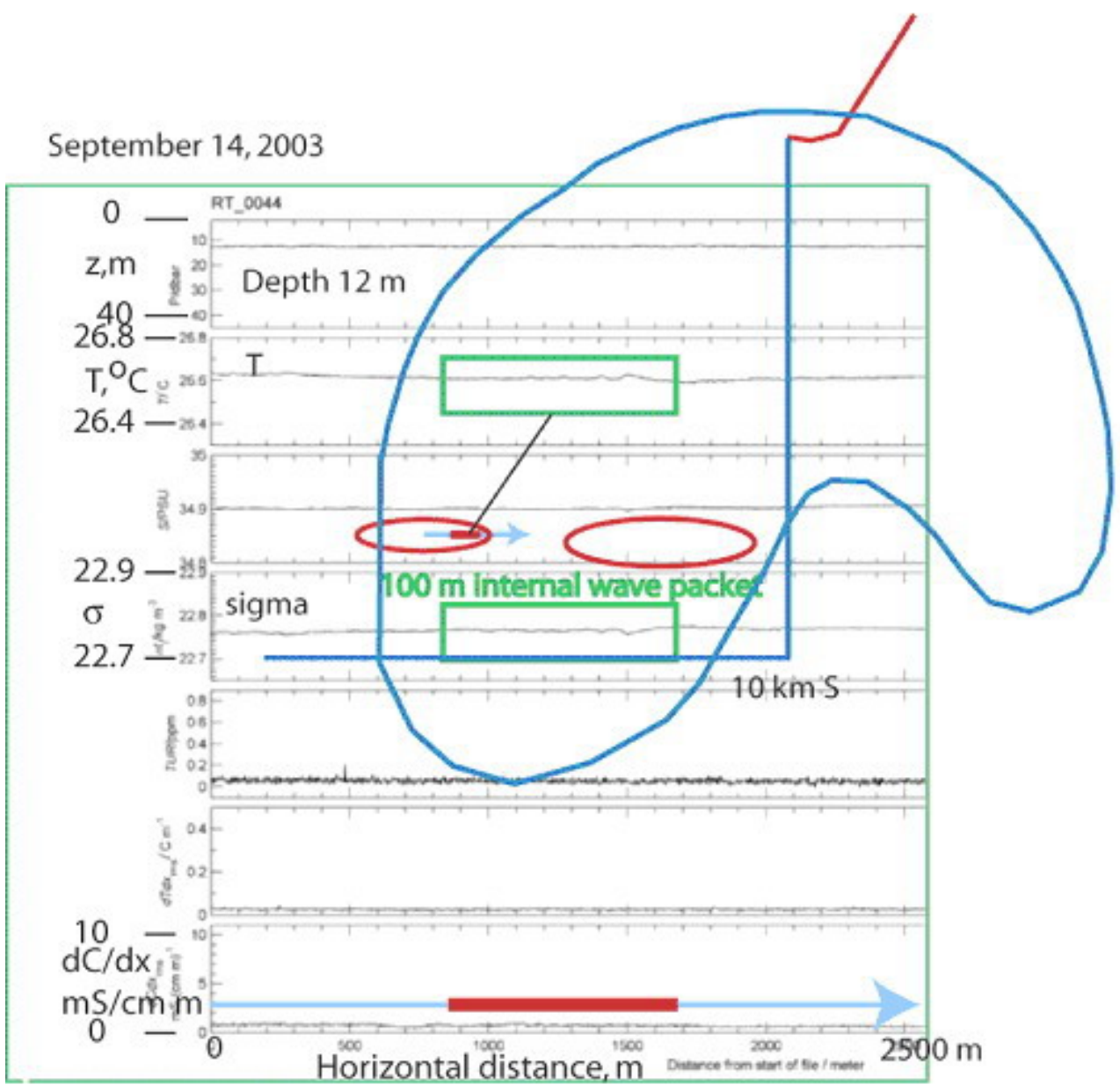

Fig. 15 - Horizontal profiles Sept. 14, 2003, $7 \mathrm{~km}$ south of the diffuser, compared to the sea surface brightness spectral anomaly pattern (Figs. 6 and 8) outlined in blue and temperature dissipation anomaly regions (mixing chimneys) in red ovals. A $900 \mathrm{~m}$ long packet of internal waves (IWs) is detected in the temperature T and potential density $\sigma\left(10^{3} \mathrm{~kg} \mathrm{~m}^{-3}\right)$ signals with $100 \mathrm{~m}$ wavelengths $\lambda$. The vertical IW displacements were about $3 \mathrm{~m}$ at a towing depth of $12 \mathrm{~m}$. The packet coincides with the western mixing chimney, Fig. 8, with scale shown by the blue arrow. 
Carl H. Gibson et al. / JAFM , Vol. 1, No. 1, pp. 11-42, 2008.
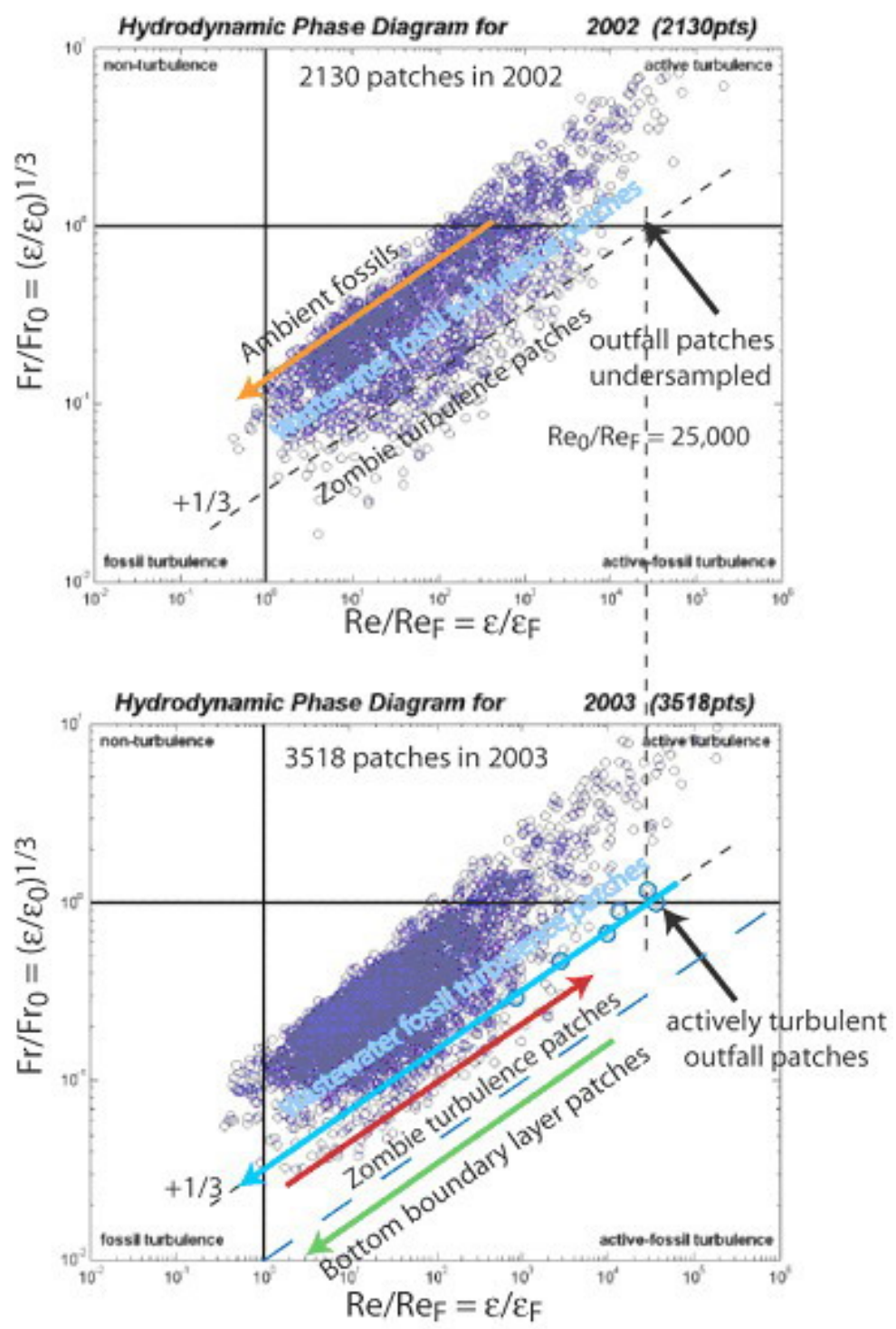

Fig. 16 - Hydrodynamic phase diagram points for all patches detected in RASP 2002 and RASP 2003 experiments. The patterns suggest the wastewater outfall produces dominant patches with $\operatorname{Re}_{0} / \operatorname{Re}_{\mathrm{F}}$ values of 25,000 significantly larger than ambient (orange arrow, $\operatorname{Re}_{0} / \operatorname{Re}_{\mathrm{F}} \sim 10^{3}$ ). Patches to the right of the $+1 / 3$ locus for this $\mathrm{Re}_{0} / \mathrm{Re}_{\mathrm{F}}$ are interpreted as zombie turbulence (red arrow, $\operatorname{Re}_{0} / \operatorname{Re}_{\mathrm{F}} \sim 10^{5}$ ). These beam information to the sea surface about the wavelength and strength of the internal waves from which they extract energy according to the BZTMA model. Deep measurements in RASP 2004 revealed fossils of BBL turbulent events with $\mathrm{Re}_{0} / \mathrm{Re}_{\mathrm{F}}$ values of $10^{6}$. 
Carl H. Gibson et al. / JAFM , Vol. 1, No. 1, pp. 11-42, 2008.

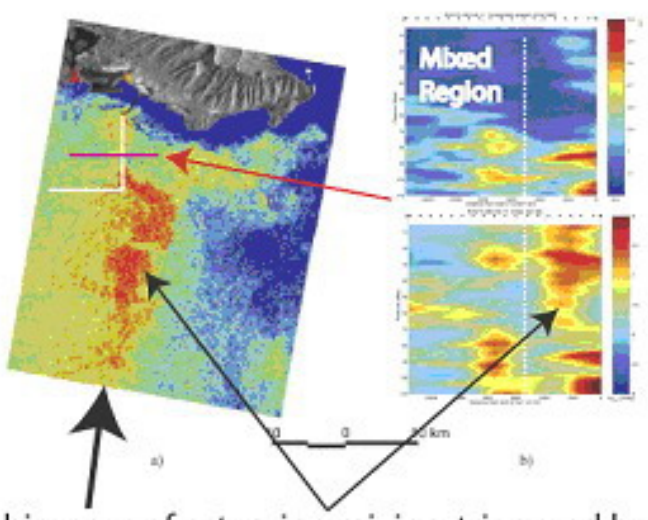

BZTMA chimneys of estuarine mixing, triggered by fossils of Sand Island outfall turbulence advected by alongshore tidal motions and strong offshore flows from Sept. 10 rains

Sept. 11, 2003 RADARSAT radar image

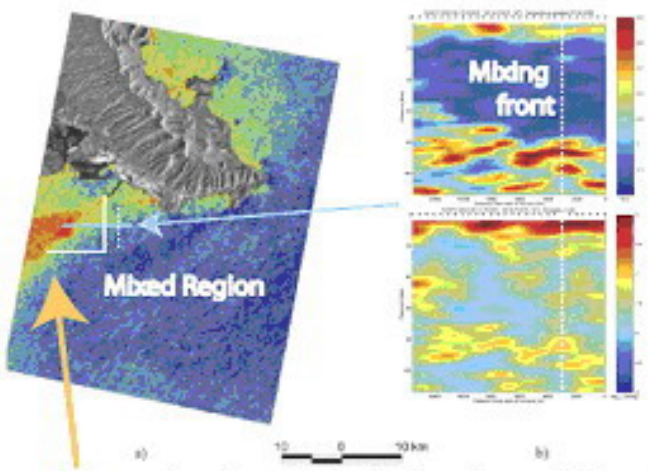

BZTMA waves from fossils of Sand Island outfall turbulence rise in mixing chimneys and break at the surface in patterns that reveal the narrow band wavelengths of the internal waves that power the zombie turbulence patches. The mechanism fails in the mixed regions.

Sept. 13, 2003 ENVISAT radar image

Fig. 17 - Radar images of the sea surface near Oahu Island on Sept. 11 (a. top) and Sept. 13, 2003 (a. bottom), compared to contour profiles from dropsonde sections of rms temperature variance (b. top-top, bottom-top) and viscous dissipation rates $\varepsilon$ (b. top-bottom, bottom-bottom). Strong rains Sept. 10, 2003, enhanced river flows so that estuarine mixing dominated the mixing on Sept. 11, preventing BZTMA detection of submerged fossil wastewater turbulence from the RADARSAT radar image. A large SW lobe brightness anomaly pattern, with about $200 \mathrm{~km}^{2}$ area was observed from the IKONOS image, and an even larger SW lobe from RADARSAT (a. bottom). The size of the SW lobe apparently reflects the large advection of wastewater turbulent fossils from Sand Island outfall shown in the high speed offshore drifter tracks of Fig. 9 for Sept. 11, reflecting the estuararine flow driven by salt wedge mixing. The strong estuarine turbulence is reflected in the radar image b. top, presumably from direct fossil turbulence wave surface smoothing, and in the lack of strong surface temperature gradient mixing and $\varepsilon$, b. top. 
Carl H. Gibson et al. / JAFM , Vol. 1, No. 1, pp. 11-42, 2008.
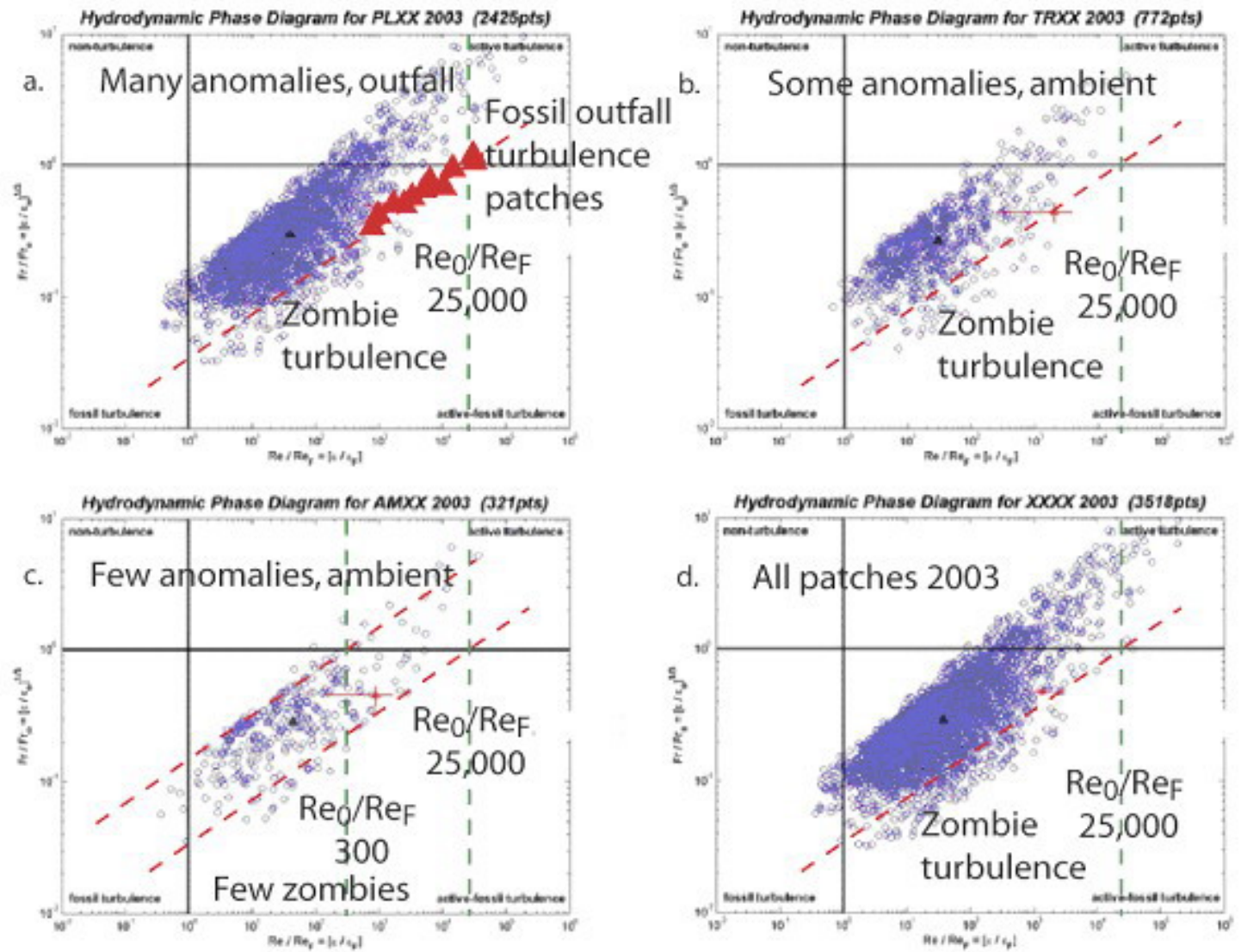

Fig. 18 - Hydrodynamic phase diagrams for RASP 2003. The patch hydrodynamic state points have been sorted to detect any differences related to the ISINTECH brightness anomalies. Patches from the regions with strong anomalies, including the outfall, are shown in a. Regions with some anomalies but excluding the diffuser are shown in b. Regions with no anomalies and excluding the diffuser are shown in $\mathbf{c}$. All regions are shown in d. 